\title{
VVV DR1: The first data release of the Milky Way bulge and southern plane from the near-infrared ESO public survey VISTA variables in the Vía Láctea ${ }^{\star}$
}

R. K. Saito ${ }^{1}$, M. Hempel ${ }^{1}$, D. Minniti 1,2,3 , P. W. Lucas $^{4}$, M. Rejkuba ${ }^{5}$, I. Toledo ${ }^{6}$, O. A. Gonzalez ${ }^{5}$, J. Alonso-García ${ }^{1}$, M. J. Irwin ${ }^{7}$, E. Gonzalez-Solares ${ }^{7}$, S. T. Hodgkin ${ }^{7}$, J. R. Lewis ${ }^{7}$, N. Cross ${ }^{8}$, V. D. Ivanov ${ }^{9}$, E. Kerins ${ }^{10}$,

J. P. Emerson ${ }^{11}$, M. Soto ${ }^{12}$, E. B. Amôres ${ }^{13,14}$, S. Gurovich ${ }^{15}$, I. Dékány ${ }^{1}$, R. Angeloni ${ }^{1}$, J. C. Beamin ${ }^{1}$, M. Catelan ${ }^{1}$, N. Padilla 1,16 , M. Zoccali ${ }^{1,17}$, P. Pietrukowicz ${ }^{18}$, C. Moni Bidin ${ }^{19}$, F. Mauro ${ }^{19}$, D. Geisler ${ }^{19}$, S. L. Folkes ${ }^{20}$, S. E. Sale ${ }^{1,20}$, J. Borissova ${ }^{20}$, R. Kurtev ${ }^{20}$, A. V. Ahumada ${ }^{9,21,22}$, M. V. Alonso ${ }^{15,21}$ A. Adamson 23 , J. I. Arias ${ }^{12}$, R. M. Bandyopadhyay ${ }^{24}$, R. H. Barbá ${ }^{12,25}$, B. Barbuy ${ }^{26}$, G. L. Baume ${ }^{27}$, L. R. Bedin ${ }^{28}$, A. Bellini $^{29}$, R. Benjamin ${ }^{30}$, E. Bica ${ }^{31}$, C. Bonatto ${ }^{31}$, L. Bronfman ${ }^{32}$, G. Carraro ${ }^{9}$, A. N. Chenè ${ }^{19,20}$, J. J. Clariá21 ${ }^{21}$ J. R. A. Clarke ${ }^{20}$, C. Contreras ${ }^{4}$, A. Corvillón ${ }^{1}$, R. de Grijs ${ }^{33,34}$, B. Dias ${ }^{26}$, J. E. Drew ${ }^{4}$, C. Fariña ${ }^{27}$, C. Feinstein ${ }^{27}$, E. Fernández-Lajús ${ }^{27}$, R. C. Gamen ${ }^{27}$, W. Gieren ${ }^{19}$, B. Goldman ${ }^{35}$, C. González-Fernández ${ }^{36}$, R. J. J. Grand ${ }^{37}$, G. Gunthardt ${ }^{21}$, N. C. Hambly ${ }^{8}$, M. M. Hanson ${ }^{38}$, K. G. Hełminiak ${ }^{1}$, M. G. Hoare ${ }^{39}$, L. Huckvale ${ }^{10}$, A. Jordán ${ }^{1}$, K. Kinemuchi ${ }^{40}$, A. Longmore ${ }^{41}$, M. López-Corredoira ${ }^{42,43}$, T. Maccarone ${ }^{44}$, D. Majaess ${ }^{45}$, E. L. Martín ${ }^{46}$, N. Masetti ${ }^{47}$, R. E. Mennickent ${ }^{19}$, I. F. Mirabel ${ }^{48,49}$, L. Monaco ${ }^{9}$, L. Morelli ${ }^{29}$, V. Motta ${ }^{20}$, T. Palma ${ }^{21}$, M. C. Parisi ${ }^{21}$, Q. Parker ${ }^{50,51}$, F. Peñaloza ${ }^{20}$, G. Pietrzyński ${ }^{18,19}$, G. Pignata ${ }^{52}$, B. Popescu ${ }^{38}$, M. A. Read ${ }^{8}$, A. Rojas ${ }^{1}$, A. Roman-Lopes ${ }^{12}$, M. T. Ruiz ${ }^{32}$, I. Saviane ${ }^{9}$, M. R. Schreiber ${ }^{20}$, A. C. Schröder ${ }^{53,54}$, S. Sharma ${ }^{20,55}$, M. D. Smith ${ }^{56}$, L. Sodré Jr. ${ }^{26}$, J. Stead ${ }^{39}$, A. W. Stephens ${ }^{57}$, M. Tamura ${ }^{58}$, C. Tappert ${ }^{20}$, M. A. Thompson ${ }^{4}$, E. Valenti ${ }^{5}$, L. Vanzi ${ }^{16,59}$, N. A. Walton ${ }^{7}$, W. Weidmann ${ }^{21}$, and A. Zijlstra ${ }^{10}$

(Affiliations can be found after the references)

Received 5 November 2011 / Accepted 22 November 2011

\section{ABSTRACT}

Context. The ESO public survey VISTA variables in the Vía Láctea (VVV) started in 2010. VVV targets 562 sq. deg in the Galactic bulge and an adjacent plane region and is expected to run for about five years.

Aims. We describe the progress of the survey observations in the first observing season, the observing strategy, and quality of the data obtained. Methods. The observations are carried out on the 4-m VISTA telescope in the $Z Y J H K_{\mathrm{s}}$ filters. In addition to the multi-band imaging the variability monitoring campaign in the $K_{\mathrm{s}}$ filter has started. Data reduction is carried out using the pipeline at the Cambridge Astronomical Survey Unit. The photometric and astrometric calibration is performed via the numerous 2MASS sources observed in each pointing.

Results. The first data release contains the aperture photometry and astrometric catalogues for 348 individual pointings in the $Z Y J H K_{\mathrm{s}}$ filters taken in the 2010 observing season. The typical image quality is $\sim 0 !^{\prime} 9-1{ }^{\prime \prime}$. 0 . The stringent photometric and image quality requirements of the survey are satisfied in $100 \%$ of the $J H K_{\mathrm{S}}$ images in the disk area and $90 \%$ of the $J H K_{\mathrm{s}}$ images in the bulge area. The completeness in the $Z$ and $Y$ images is $84 \%$ in the disk, and $40 \%$ in the bulge. The first season catalogues contain $1.28 \times 10^{8}$ stellar sources in the bulge and $1.68 \times 10^{8}$ in the disk area detected in at least one of the photometric bands. The combined, multi-band catalogues contain more than $1.63 \times 10^{8}$ stellar sources. About $10 \%$ of these are double detections because of overlapping adjacent pointings. These overlapping multiple detections are used to characterise the quality of the data. The images in the $J H K_{\mathrm{s}}$ bands extend typically $\sim 4$ mag deeper than 2MASS. The magnitude limit and photometric quality depend strongly on crowding in the inner Galactic regions. The astrometry for $K_{\mathrm{s}}=15-18$ mag has rms $\sim 35-175$ mas.

Conclusions. The VVV Survey data products offer a unique dataset to map the stellar populations in the Galactic bulge and the adjacent plane and provide an exciting new tool for the study of the structure, content, and star-formation history of our Galaxy, as well as for investigations of the newly discovered star clusters, star-forming regions in the disk, high proper motion stars, asteroids, planetary nebulae, and other interesting objects.

Key words. Galaxy: bulge - Galaxy: disk - Galaxy: stellar content - stars: abundances - infrared: stars - surveys

\section{Introduction}

The VISTA variables in the Vía Láctea (VVV) survey is mapping 562 square degrees in the Galactic bulge and the southern disk in the near-infrared (Minniti et al. 2010). The VVV survey gives near-infrared (near-IR) multi-colour information in five

$\star$ Based on observations taken within the ESO VISTA Public Survey VVV, Programme ID 179.B-2002. passbands: $Z(0.87 \mu \mathrm{m}), Y(1.02 \mu \mathrm{m}), J(1.25 \mu \mathrm{m}), H(1.64 \mu \mathrm{m})$, and $K_{\mathrm{s}}(2.14 \mu \mathrm{m})$, as well as time coverage spanning over five years, which will complement past/recent, current, and upcoming surveys such as 2MASS, DENIS, GLIMPSE-II, VPHAS+, MACHO, OGLE, EROS, MOA, and GAIA.

VVV is an ESO Public Survey, i.e., the observational raw data are made available to the astronomical community immediately, whereas the reduced data will be published once a year 
in a data release through ESO. This paper describes and characterises the first data release (DR1) of the VVV survey. Some first results of the VVV survey based on early science images taken in the bulge and disk fields are highlighted in Saito et al. (2010) and Catelan et al. (2011).

The preparatory phase for the VVV survey started in 2006, with the first test observations obtained in October 2009. Regular operations started with the first survey observations in February 2010. The data collected during the first year of observations until October 2010 are the subject of this public release. Our survey is planned to be carried out for five years, and we expect to produce yearly accumulated data releases.

The VVV survey is foremost a variability study of the inner regions of the Milky Way (Minniti et al. 2010), but will also complement the existing 2MASS $J H K$ photometry (Cutri et al. 2003), extending to much fainter limits while adding two additional filters $(Z Y)$, and providing time domain information useful for variability and proper motion studies. In particular, the higher resolution of the VVV data represents a huge advantage in crowded fields compared to previous near-IR surveys such as 2MASS and DENIS (Epchtein et al. 1994), where the single-epoch photometry was confusion-limited, reaching $K_{\mathrm{s}} \sim$ 14.3 mag. The limiting magnitude of the VVV data using aperture photometry is $K_{\mathrm{s}} \sim 18.0 \mathrm{mag}$ in most fields. Even in the innermost fields $\left(|b| \leq 1^{\circ}\right)$ the VVV survey reaches $K_{\mathrm{s}} \sim 16.5 \mathrm{mag}$, at least a magnitude deeper than the IRSF/SIRIUS survey of the Galactic Centre (Nagayama et al. 2003; Nishiyama et al. 2006, 2009).

The VVV survey was designed to complement the UKIDSSGPS (Lucas et al. 2008), VPHAS+ (see Arnaboldi et al. 2007), and the GLIMPSE-II surveys (Benjamin et al. 2003). The UKIDSS-GPS is mapping $|b|<5^{\circ}$ in Galactic latitude in the northern plane for three epochs, while VPHAS+ also observes the Galactic plane in the optical and $\mathrm{H} \alpha$ using the ESO VLT Survey Telescope (VST).

The GLIMPSE-II survey images the central $\pm 10^{\circ}$ of the plane in four bands with IRAC. VVV provides variability information for the overlap region, supporting studies of the content and distribution of stars, stellar populations, and interactions of the strong nuclear wind with the ambient of the interstellar medium (ISM) above and below the nucleus, as well as the rate and location of current star formation.

Multiband Spitzer public surveys with IRAC (mid-IR at $3.6 \mu \mathrm{m}, 4.5 \mu \mathrm{m}, 5.6 \mu \mathrm{m}$ and $8.0 \mu \mathrm{m}$ ) and MIPS (far-IR, $23.7 \mu \mathrm{m}$, $71.4 \mu \mathrm{m}$ weighted average wavelength), respectively, cover the mid-plane $\left(|b|<1^{\circ}\right)$ at $65^{\circ}<l<10^{\circ}$ and $-10^{\circ}<l<-65^{\circ}$. The southern half of these surveys overlaps with the VVV disk area, allowing the detection and characterisation of star-formation regions and probing the structure of the inner disk of the Galaxy. In optically obscured regions the IRAC data complement the VVV survey and those of VST/VPHAS+ by tracing the influence of the most massive stars on star formation.

In addition, we complement the existing bulge microlensing surveys such as OGLE (Udalski et al. 1993; Szymański et al. 2011) and MACHO (Alcock et al. 2000), which observe in optical bands, with limited or no colour information. These surveys mostly concentrate on regions of low extinction. VVV will provide useful variability information for the overlap regions.

The data are of excellent quality in general, and only a small fraction did not pass our quality controls and had to be reacquired. These DR1 data have passed all the initial quality controls as performed by the survey team in collaboration with the
Cambridge Astronomical Survey Unit $(\mathrm{CASU})^{1}$. We checked image defects, telescope problems, seeing, zero point, magnitude limit, ellipticity, and airmass, to name a few. However, it is important to stress that the data quality and calibrations will improve with subsequent data releases.

Here we will address the general information for the community, e.g., on the survey area and strategy, data quality, progress in the observations, published source lists, as well as examples of specific applications. In addition, the data, procedures, and additional information are available through the ESO archive ${ }^{2}$, the VVV survey science team homepage ${ }^{3}$, CASU, and through the VISTA science archive (VSA) webpage ${ }^{4}$.

This paper is organized as follows: Sect. 2 describes the area coverage and the observations with VISTA as well as the data processing. Section 3 describes the photometric quality (limits, accuracy), and completeness. Section 4 discusses a comparison with 2 MASS. Section 5 presents a comparison between the VVV DR1 (aperture) catalogues and point spread function (PSF) photometry. Section 6 describes the astrometric data quality. Section 7 presents density maps for the bulge and disk fields. Section 8 describes a suitability test of the DR1 data for difference image analysis.

The final section summarizes and presents our conclusions, including important caveats regarding this DR1, and future improvements to be implemented in DR2. Finally, the VVV tile coordinates are listed in the appendix.

\section{Survey area and observations}

\subsection{Telescope and instrument}

The telescope used to carry out the VVV survey is VISTA (Visible and Infrared Survey Telescope for Astronomy), a 4-m class wide-field telescope with a single instrument, VIRCAM (VISTA InfraRed CAMera; Dalton et al. 2006; Emerson \& Sutherland 2010), located on its own peak at ESO's Cerro Paranal Observatory in Chile, about $1500 \mathrm{~m}$ away from the VLT. Its primary mirror has a diameter of $4.1 \mathrm{~m}$, providing a $f / 3.25$ focal ratio at the Cassegrain focus where the instrument is mounted. The secondary mirror has a $1.24 \mathrm{~m}$ diameter. The start of survey operations of the telescope was on April 1, 2010, but most VISTA surveys, including VVV, started collecting science observations a few months earlier, in parallel with the last phases of the scientific performance verification and operations fine-tuning performed by Paranal Observatory staff. During the first year of operations the mirrors were coated with silver, which is optimized for near-IR observations.

With $1.64 \mathrm{deg}$ diameter VIRCAM offers the largest unvignetted field of view in the near-IR regime on 4-m class telescopes. It is equipped with 16 Raytheon VIRGO $2048 \times$ 2048 pixels $^{2}$ HgCdTe science detectors, with 0.339 average pixel scale. Each individual detector therefore covers $\sim 694 \times$ $694 \operatorname{arcsec}^{2}$ on the sky. The achieved image quality (including seeing) is better than $\sim 0$ ' 6 on axis. The image quality distortions are up to about $10 \%$ across the wide field of view. The detectors are arranged in a $4 \times 4$ array, with large spacings of $90 \%$ and $42.5 \%$ of the detector size along the $X$ and $Y$ axes, respectively. A single pointing, called a pawprint, covers 0.59 sq. deg, and provides partial coverage of the field of view. By combining six

\footnotetext{
1 http://casu.ast.cam.ac.uk/vistasp/

2 http://www. eso.org/sci/archive.html

3 http://vvvsurvey.org

4 http://horus.roe.ac.uk/vsa/index.html
} 


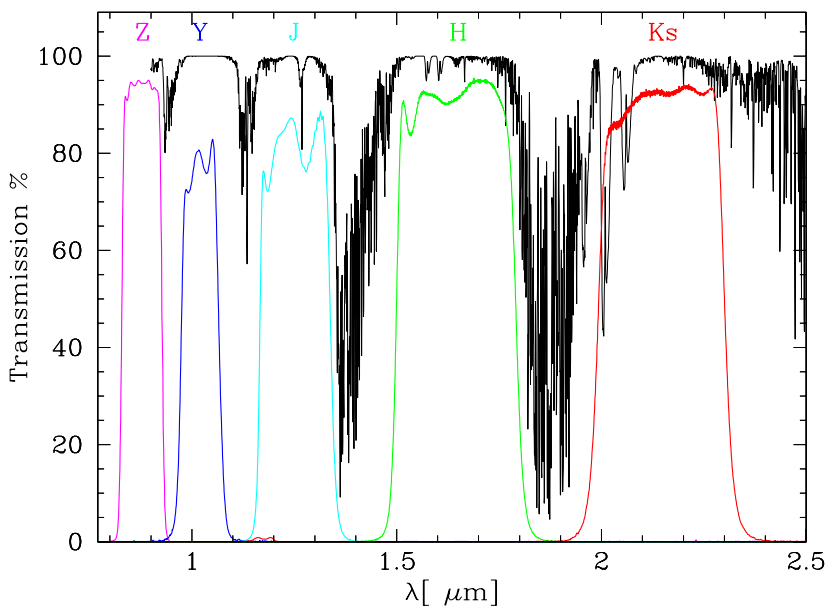

Fig. 1. Transmission curves for the five broad-band filters present at the VIRCAM: $Z, Y, J, H$ and $K_{\mathrm{s}}$, compared with the typical atmospheric transmission profile for airmass $=1.0$ and $1.0 \mathrm{~mm}$ water vapour. The effective wavelengths for all filters are listed in Table 1.

Table 1. Effective wavelengths for the VISTA filter set used in the VVV observations and the relative extinction for each filter based on the Cardelli et al. (1989) extinction law (from Catelan et al. 2011).

\begin{tabular}{lccc}
\hline \hline Filter & $\lambda_{\text {eff }}(\mu \mathrm{m})$ & $A_{X} / A_{V}$ & $A_{X} / E(B-V)$ \\
\hline$Z$ & 0.878 & 0.499 & 1.542 \\
$Y$ & 1.021 & 0.390 & 1.206 \\
$J$ & 1.254 & 0.280 & 0.866 \\
$H$ & 1.646 & 0.184 & 0.567 \\
$K_{\mathrm{S}}$ & 2.149 & 0.118 & 0.364 \\
\hline
\end{tabular}

pawprint exposures with appropriate offsets, a contiguous coverage of a field is achieved with at least two exposures per pixel except at two edges. In all VISTA observations this field is called a tile and covers a 1.64 sq. deg field of view. Throughout this paper we will use a tile as the individual exposure.

VIRCAM has four additional optical CCDs, two for guiding and two for active optics. For exposures longer than $\sim 40 \mathrm{~s}$ the active optics is run in parallel mode with the observations. Owing VVV's very short individual exposures (see Sect. 2.3) the active optics correction is only performed every $\sim 30$ min or after a larger offset. This, combined with the need to survey the area fast (hence minimizing the overheads for more frequent active optics corrections), and typical seeing on Paranal limits the image quality obtained for the survey data to typically $\sim 0.9-1.0 \operatorname{arcsec}$ (see Sect. 2.4).

VIRCAM is equipped with five broad-band filters $(Z, Y$, $J, H$, and $K_{\mathrm{s}}$ ) and two narrow-band filters centred at 0.98 and $1.18 \mu \mathrm{m}$. The VVV survey uses all five broad-band filters spanning from 0.84 to $2.5 \mu \mathrm{m}$. Their effective wavelengths and relative extinctions are given in Table 1, while the transmission curves are shown in Fig. 1, compared to a typical atmospheric transmission profile for airmass 1.0 and $1 \mathrm{~mm}$ water vapour in the atmosphere.

For more details about the telescope and instrument we refer the interested reader to the VIRCAM instrument web pages ${ }^{5}$, and the VISTA/VIRCAM user manual (Ivanov \& Szeifert 2009).

\subsection{Survey area}

The VVV survey area consists of 348 tiles, 196 tiles in the bulge and 152 in the disk area. These two components were planned to

\footnotetext{
5 http://www.eso.org/sci/facilities/paranal/ instruments/vircam/
}

Table 2. VVV survey completion in the 2010 season.

\begin{tabular}{lccc}
\hline \hline $\begin{array}{l}\text { Tile } \\
\text { type }\end{array}$ & $\begin{array}{c}\text { Completed } \\
\text { Tile }\end{array}$ & $\begin{array}{c}\text { Total } \\
\text { Tiles }\end{array}$ & $\begin{array}{c}\text { Completion } \\
\text { percentage }\end{array}$ \\
\hline \multicolumn{4}{c}{ Bulge } \\
$J H K_{\mathrm{s}}$ & 188 & 196 & $95 \%$ \\
$Z Y$ & 78 & 196 & $40 \%$ \\
Variability & 113 & $5 \times 196$ & $12 \%$ \\
\hline \multicolumn{4}{c}{ Disk } \\
$J H K_{\mathrm{s}}$ & 152 & 152 & $100 \%$ \\
$Z Y$ & 128 & 152 & $84 \%$ \\
Variability & 547 & $5 \times 152$ & $72 \%$ \\
\hline
\end{tabular}

cover 520 sq. deg, as follows: (i) the VVV bulge survey area covers 300 sq. deg between $-10^{\circ} \leq l \leq+10^{\circ}$ and $-10^{\circ} \leq b \leq+5^{\circ}$; and (ii) the VVV disk survey area covers 220 sq. deg between $295^{\circ} \leq l \leq 350^{\circ}$ and $-2^{\circ} \leq b \leq+2^{\circ}$. However, to maximize the efficiency of the tilling process (see Sect. 2.3), the survey area definition tool (SADT; Hilker et al. 2011) produced some shifts at the edges of the survey area, and as the result an area of $\sim 562$ sq. deg (42 sq. deg larger) was observed. Thus, the observed area is within $-10.0^{\circ} \lesssim l \lesssim+10.4^{\circ}$ and within $-10.3^{\circ} \lesssim b \lesssim+5.1^{\circ}$ in the bulge, and $294.7^{\circ} \lesssim l \lesssim 350.0^{\circ}$ and $-2.25^{\circ} \lesssim b \lesssim+2.25^{\circ}$ in the disk. The VVV survey area and tile numbering are shown in Fig. 2, while the list of all tile centres in equatorial and Galactic coordinates is given in Table A.1. The tile names start with "b" for bulge and "d" for disk tiles, followed by the numbering shown in Fig. 2 .

While the whole area was observed in the $J H K_{\mathrm{s}}, 95 \%$ of these tiles satisfy the stringent photometric and image quality parameters and are classified as completed. In the $Z Y$ bands the completion is somewhat lower, with $59 \%$ completed tiles. The tiles completed in the first season (until October 26, 2010) can be seen in Figs. $3-5$ for $Z Y, J H$, and $K_{\mathrm{s}}$, respectively. Figure 5 also includes the tiles with at least one epoch observed during the variability campaign in the $K_{\mathrm{s}}$ band.

In the first season of the variability campaign 22 tiles in the disk area had five $K_{\mathrm{s}}$ epochs taken, while the majority had one or more additional $K_{\mathrm{s}}$ epochs completed. The completion rates individually for the bulge and disk areas are given in Table 2. Table A.1 states for each tile whether the observations in a given filter are completed, and how many additional $K_{\mathrm{s}}$ epochs were obtained until October 26, 2010.

Figure 6 shows cumulative distributions of image quality and airmass for the observed tiles in the 2010 season. The median image quality in the $J, H$ and $K_{\mathrm{s}}$ filters is better than $0 !^{\prime} 9$ as measured on combined tile images, while it is close to 1 .' 0 for the $Z$ and $Y$ filters.

\subsection{Observing strategy}

The first observations collected for the VVV survey were taken during the VISTA science verification period in October 2009 when one field in the Galactic bulge at $\alpha=18: 02: 58.872$, $\delta=-28: 36: 59.04$ (J2000) was observed in the $Z Y J H K_{\mathrm{s}}$ filters (called SV field). In addition to the nearly-simultaneous multiband images of the SV field, $11 K_{\mathrm{S}}$ band exposures were taken to test the variability observing strategy. To establish the necessary number of exposures for proper sky subtraction, depending on crowding and number of resolved objects in the field, three additional tiles were observed in the $K_{\mathrm{s}}$ band, two bordering directly on the SV field and one at an offset position $\approx 1^{\circ}$ south of the science target. Based on these early observations, we adjusted 


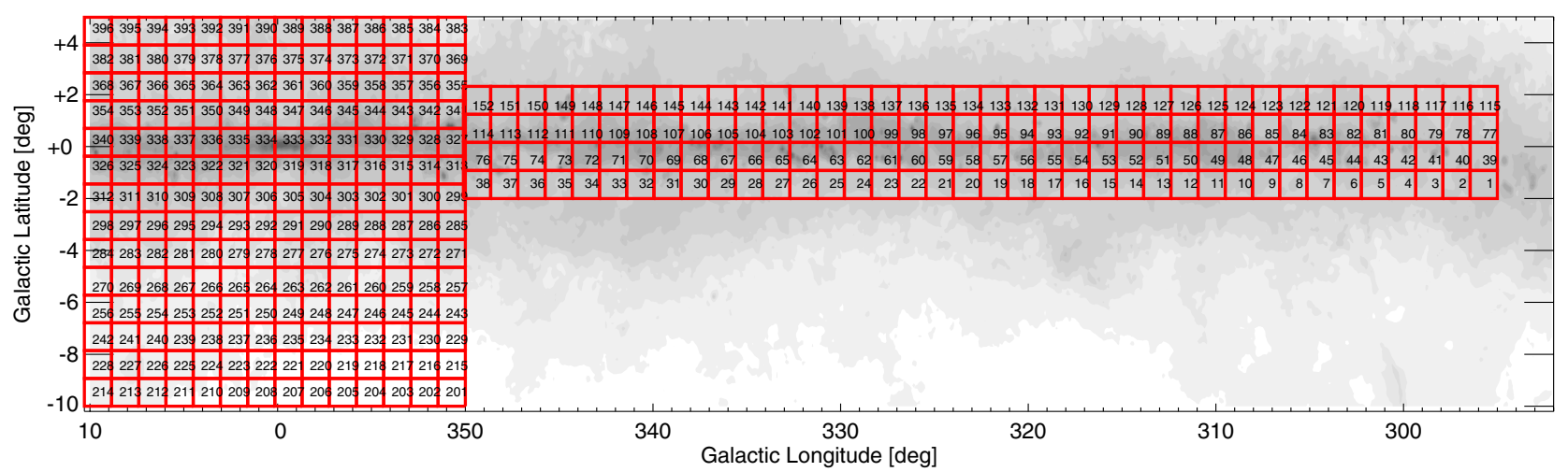

Fig. 2. VVV survey area and tile numbering. The tile names start with "b" for bulge and "d" for disk tiles, followed by the numbering as shown in the figure. The centre coordinates for all VVV tiles are listed in Table A.1.
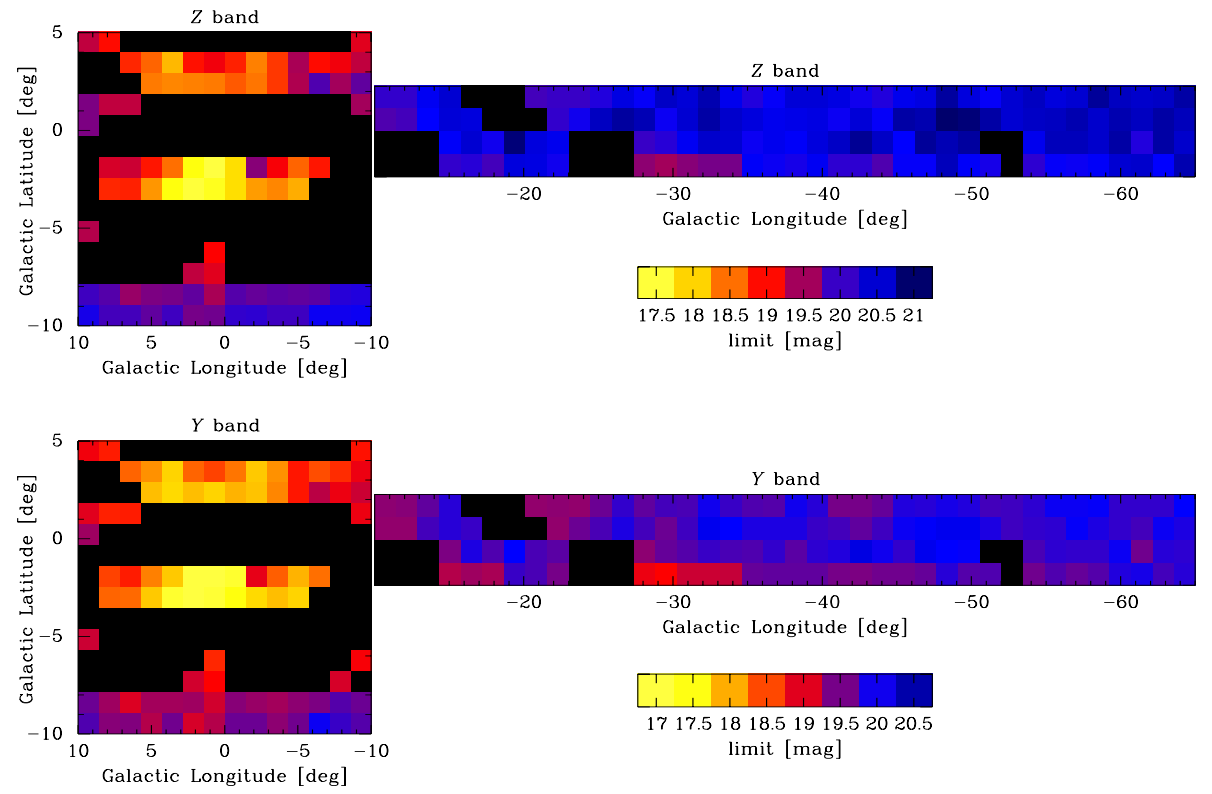

Fig. 3. $5 \sigma$ magnitude limits of the catalogues in the $Z$ - (top panel) and $Y$-bands (bottom panel). The colour scale is shown in each case. The completeness of the DR1 can be also checked on the maps, where the missing tiles appear in black. Exposure times in the bulge and disk fields are different (see Table 3), as a result the disk fields have deeper photometry. Similar maps for $J, H$ and $K_{\mathrm{s}}$ photometry, and for the variability campaign are presented in Figs. 4 and 5.

Table 3. Observing strategy and exposure times for VVV OBs.

\begin{tabular}{lcccc}
\hline \hline Area & Filter & $\begin{array}{c}\text { DIT }^{a} \\
(\mathrm{~s})\end{array}$ & NDIT $^{b}$ & $\begin{array}{c}\text { Median exp. time } \\
\text { per pixel (s) }\end{array}$ \\
\hline Bulge & $Z, Y$ & 10 & 1 & 40 \\
Bulge & $J$ & 6 & 2 & 48 \\
Bulge & $H, K_{\mathrm{s}}$ & 4 & 1 & 16 \\
Bulge & $K_{\mathrm{s}}(\mathrm{var})$ & 4 & 1 & 16 \\
\hline Disk & $Z, Y$ & 20 & 1 & 80 \\
Disk & $J, H, K_{\mathrm{s}}$ & 10 & 2 & 80 \\
Disk & $K_{\mathrm{s}}(\mathrm{var})$ & 4 & 1 & 16 \\
\hline
\end{tabular}

Notes. ${ }^{(a)}$ Detector integration time; ${ }^{(b)}$ number of DITs.

the observing strategy, which differs slightly between the bulge and disk fields (i.e., with respect to exposure time, number of coadded exposures, and combination of tiles for sky subtraction).

Like all VISTA observations, the VVV survey is carried out in service mode. The basic observational unit is the so-called OB (observation block). The multi-filter, single-epoch OBs have been split into $J H K_{\mathrm{s}}$ and $Z Y$ OBs. The variability monitoring OBs have only single filter: $K_{\mathrm{s}}$. Observation blocks for two, three or four tiles were executed back-to-back to ensure that a sufficient number of offset images were taken for each filter to create a high-quality background sky frame.

The survey area was defined with the help of the SADT, which provides the tile centres as well as guide and active optics stars that are necessary for the efficient execution of the survey OBs. Apart from the edges of the survey area, the input to SADT is also the tile pattern, which defines the large offsets that fill in the inter-detector gaps, and the size of the smaller (jitter) offsets that are executed at each of the six pawprint positions that together make a tile. All VVV OBs used the "Tile6n" pattern. In addition, at each of the six pawprint positions two smaller offsets are executed using the "Jitter $2 u$ " pattern. This means that in total there are 12 exposures per filter, but given the large offsets, each pixel is covered by at least four exposures, except for the pixels along the $y$-edge of the tile (two jitter positions and at least two pawprints). These, however, have overlaps with adjacent tiles. Therefore the complete survey area is covered by at least four exposures in each filter. Each image is a co-addition 

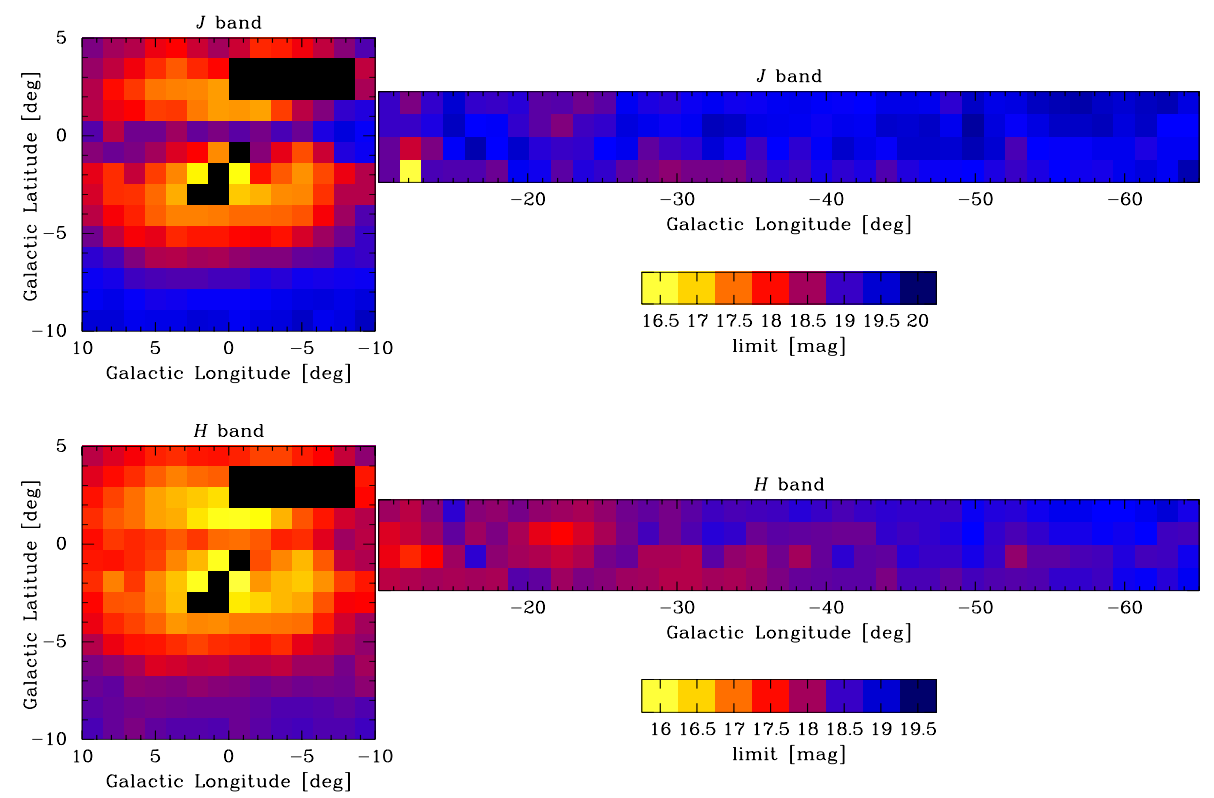

Fig. 4. $5 \sigma$ magnitude limits of the catalogues in the $J$ - (top panel) and $H$-bands (bottom panel). The notation is similar to that presented in Fig. 3 .
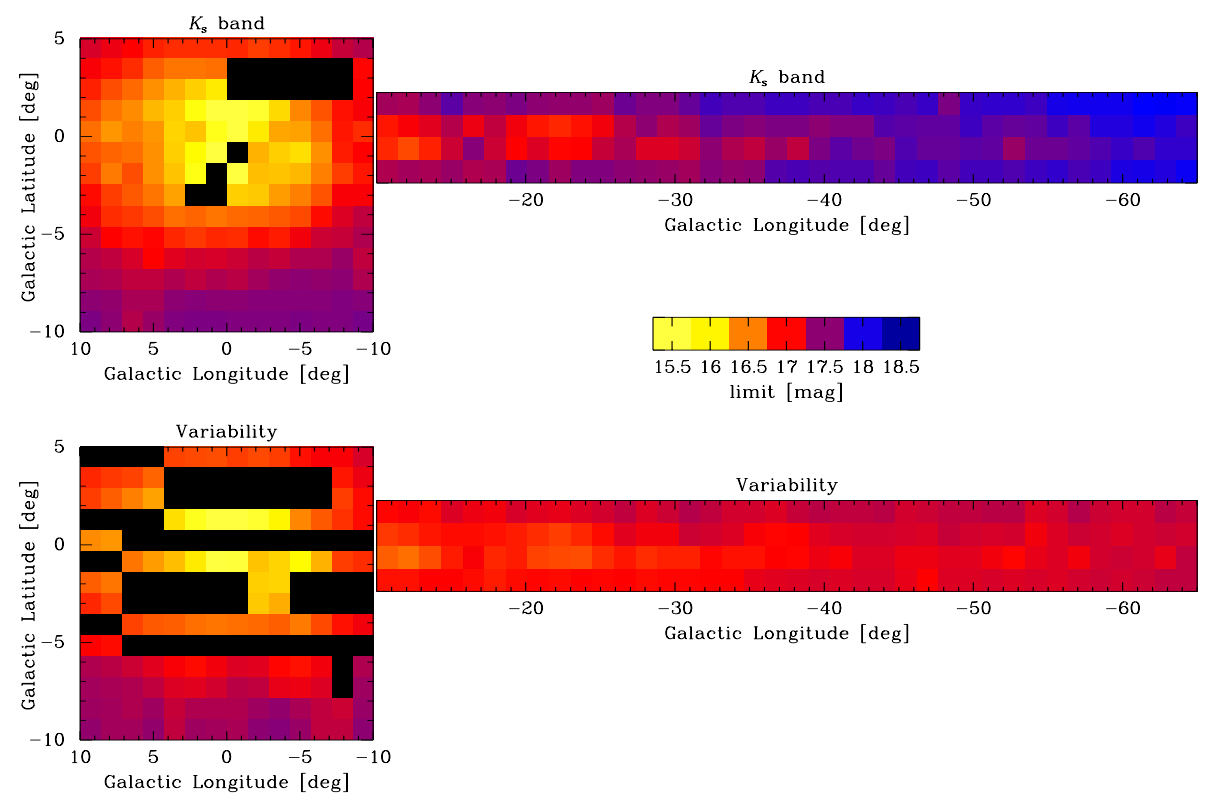

Fig. 5. $5 \sigma$ magnitude limit of the catalogues in the $K_{\mathrm{s}}$ band (top panel) and for the variability campaign (also performed in the $K_{\mathrm{s}}$ band, bottom panel). The colour scale is the same in both maps. Different strategies between the regular $K_{\mathrm{s}}$ observations and the variability campaign causes that in disk fields the variability data show shallower photometry. The notation is similar to that presented in Fig. 3.

of NDIT (number of detector integration time) exposures lasting DIT (detector integration time) seconds each. The total exposure times for bulge and disk tiles are given in Table 3.

\subsection{Data processing}

The VVV observations were pipeline-processed within the VISTA data flow system (VDFS) pipeline at the CASU (Lewis et al. 2010). The processing was performed on a night-by-night basis, and consisted of the following data reduction steps executed in the order described.

The mean dark current exposure, taken with the same DIT and NDIT values, is subtracted from each image.

A linearity correction is applied for individual detectors using information on the readout time, exposure time, and the reset image time. A "reset" exposure of $1.0 \mathrm{~s}$ for every exposure is subtracted from each exposure within the data acquisition system, prior to writing the image to the disk.

The flat-field correction is made by dividing by a mean twilight flat-field image to remove small-scale quantum efficiency variations and the large-scale vignetting profile of the camera, as well as to normalize the gain of each detector to a common median value.

The sky background correction removes the large-scale spatial background emission. Tests made with the science verification observations showed that 12 exposures (six pawprint $\times$ two jitters) taken for each VVV tile do not yield good sky subtraction because of severely crowded fields, leaving "holes" at the positions of bright stars or very crowded regions with many overlapping stellar PSFs. Therefore a sky background map is 

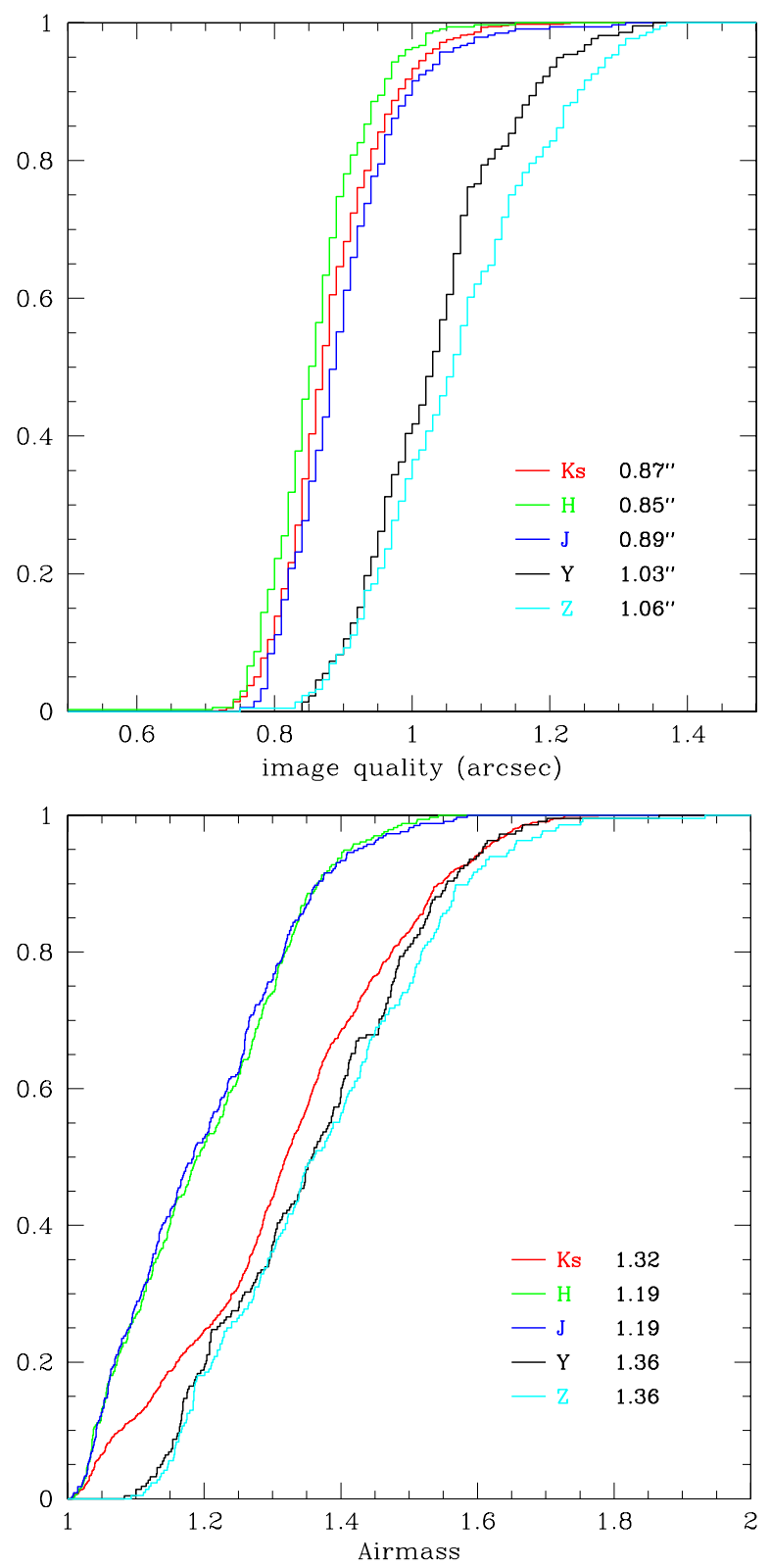

Fig. 6. Image quality and airmass cumulative distributions for the VVV observations obtained in 2010 in the $Z Y J H K_{\mathrm{s}}$ filters are plotted in the top and bottom panels, respectively. The median values of image quality and airmass for each filter are given in the legends.

produced for VVV by combining all exposures for a given filter taken within several concatenated OBs (tiles). Owing to variability of the near-IR sky, the need to take at least 24 images with the same filter within $\sim 30$ min poses limits to the exposure times for individual tiles and the number of different filters that can be included in each OB.

A "destriping correction" is performed by subtracting the low-level horizontal stripe pattern introduced by the readout electronics of the VIRCAM detectors.

Jitter stacking is performed to align two slightly shifted images taken at a given pawprint position, combining them into a single image for each pawprint. The shifts are computed using the positions of many hundreds of stars detected in all images.

Object detection is performed for each stacked pawprint image. Positions, fluxes measured in several apertures of different sizes, and some shape measurements are written in the source catalogue. A flag indicates the most probable morphological classification, and in particular we note that " -1 " is used to denote stellar objects, " -2 " borderline stellar, " 0 " is noise, and " +1 ' is used for non-stellar objects. There are also objects with flag "-7", denoting sources containing bad pixels, and the flag "-9" is used for saturated stars. These flags are derived mainly based on curve-of-growth analysis of the flux (Irwin et al. 2004). Figure 7 shows colour-magnitude diagrams (CMDs) for a moderately crowded bulge field (b264), comparing the distribution of the high-quality sources with all other flags. Relative numbers for each flag are also indicated in the figure. In addition to a catalogue with extracted sources, a confidence map is also computed (see Fig. 8).

The six stacked pawprint images are combined into a single deep tile and the catalogue extraction step is repeated. Tile images contiguously sample about $1.5 \times 1.1 \mathrm{sq}$. deg on the sky. Confidence maps are computed for each tile, relaying information on the different exposure times for pixels across the tile (Fig. 8). The exposure times depend on the size of the jitter offsets and the number of exposures that are combined into a tile. The confidence maps clearly show the areas of detectors affected by bad pixels, such as the large patch in detector 1 (lower left corner in the right panel of Fig. 8), several rows in detector 4 (lower right corner) and the upper third of detector 16 (upper right corner). After stacking several dithered exposures in the final tile, most of these bad pixels are not noticeable (left panel of Fig. 8), but the large bad area on detector 16 has larger errors in the illumination correction map. The sensitivity of that upper third of the detector is much worse at shorter wavelengths, and some offsets with respect to 2MASS calibration for the whole tile have been found in $Z, Y$ and $J$ bands. Offsets at longer wavelengths in the $H$ and $K_{\mathrm{s}}$ bands are within the calibration errors.

The first-year data release of the tile images, catalogues, and confidence maps described here is version 1.1. Detailed information about this version of VISTA data products as well as a brief description of all problems encountered during data processing is available on the CASU web page ${ }^{6}$.

\subsubsection{Generation of multi-band catalogues}

Currently the version 1.1 single-band tile catalogues from CASU are matched by the VVV team members using the STILTS package (Taylor 2006) and a KD-Tree based algorithm (Gurovich et al., in prep.) that uses the Cross et al. (in prep.) implemented source matching method for the VSA data. They are matched using astrometrically corrected tiles and catalogues, allowing a 1". 0 offset between point sources to be considered a match. Nevertheless, tests have shown that over $90 \%$ of the stellar sources are matched within less than 0.5 , as expected from the astrometric accuracy of the catalogues (see Sect. 6).

After matching the single-band catalogues, most of the spurious detections around bright stars are rejected. Unfortunately, the ellipticity sometimes varies from image to image; which then results in rejection of some sources which are only classified as "bona fide" stars in selected filters, but appear slightly more elongated, and are therefore rejected as non-stellar in other filters. A final multi-band catalogue contains $\sim 75-85 \%$ of the sources found in the catalogue with the least number of sources used for the matching, in general the $K_{\mathrm{s}}$ band, for lowto intermediate-extinction regions. Close to the Galactic plane, high extinction affects the source detection at short wavelengths

\footnotetext{
${ }^{6}$ http://casu.ast.cam.ac.uk/surveys-projects/vista/ technical
} 


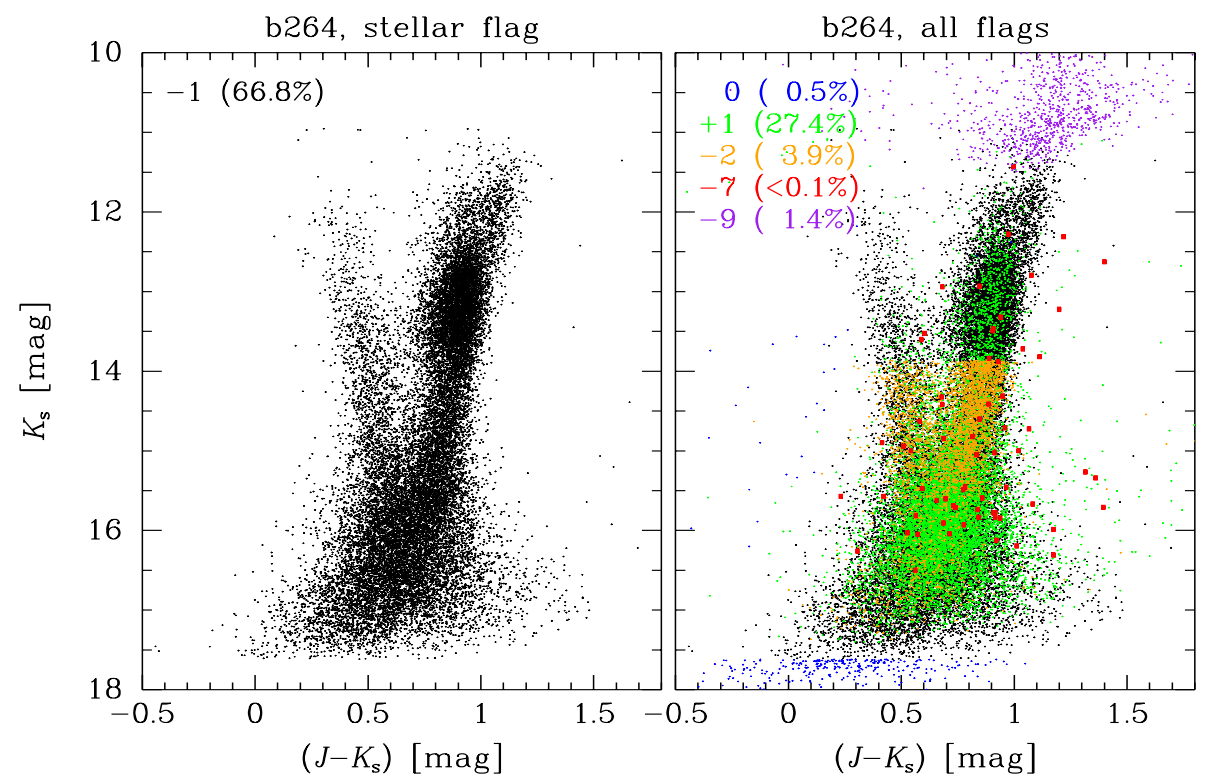

Fig. 7. $K_{\mathrm{s}}$ vs. $\left(J-K_{\mathrm{s}}\right)$ CMDs for a moderately crowded bulge field (a section of tile b264), showing the high-quality sources with -1 , stellar flag (left panel), compared to all other flags found in the CASU catalogues (right panel). The relative number of sources is given in the top left corner.

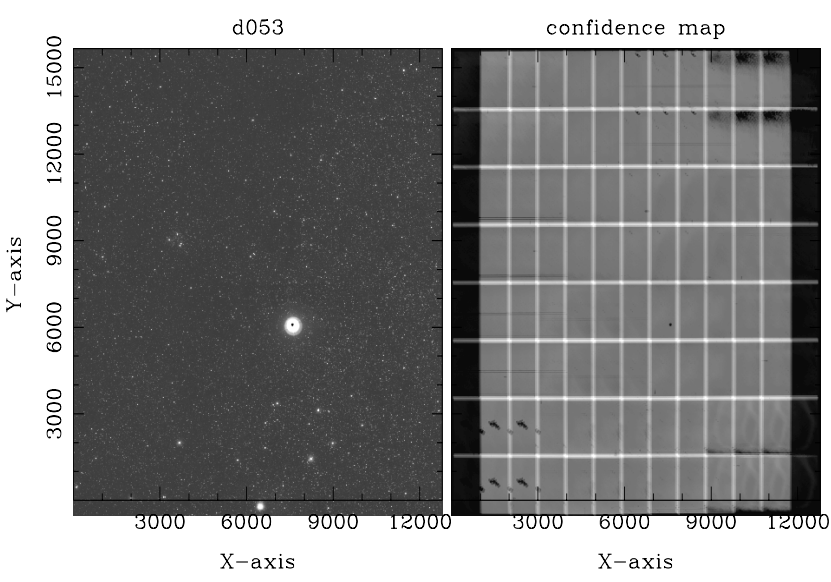

Fig. 8. Left panel: the disk tile d053 in the $K_{\mathrm{s}}$ band with its corresponding confidence map shown in the right panel. The brighter regions in the confidence map have a longer total exposure time, owing to the combination of the six pawprints. This image also includes two jitters at each pawprint position. Clearly visible in the lower left corner is a cluster of bad pixels (Chip 1). The six different positions correspond to the six pawprints, which are combined to fill the gaps between individual detectors.

more severely, hence the source density is limited by the detection rate in the $Y$ and $Z$ bands. The comparison between the different tiles is hampered not only by the different median extinction values, but also by the strong extinction variation within any given tile. As an example, we divided two tiles (b305 and $\mathrm{d} 003$ ) into $0.25^{\circ} \times 0.25^{\circ}$ sub-sections. Median extinction values $A_{\mathrm{V}}$ and their standard deviations are given in Table 4.

Multi-band catalogues corresponding to the DR1 data have also been generated by the VISTA Science Archive (VSA). While the single-band DR1 data were already delivered through the ESO archive, the multi-band catalogues will be publicly available in due time through the ESO archive and VSA web page $^{7}$. The VSA will also provide multi-epoch catalogues for variable sources. Part of the data reduction and storage are performed using the Geryon cluster at the Center for AstroEngineering at Universidad Católica (AIUC) ${ }^{8}$.

\footnotetext{
7 http://horus.roe.ac.uk/vsa/index.html

8 http://www.aiuc.puc.cl/
}

Table 4. Galactic extinction values (median) for tiles b305 and d003, assuming $A_{V}=3.1 \times E(B-V)$ and based on the prescriptions by (1) Schlegel et al. (1998); (2) Drimmel et al. (2003); (3) Amôres \& Lépine (2005); (4) Marshall et al. (2006); (5) Froebrich et al. (2005); (6) Dobashi et al. (2005).

\begin{tabular}{lccc}
\hline \hline Tile & Reference & $A_{V}($ median $)$ & $\sigma\left(A_{V}\right)$ \\
\hline b305 & $(1)$ & 4.04 & 1.54 \\
& $(2)$ & 4.81 & 1.81 \\
& $(3)$ & 3.11 & 0.67 \\
& $(4)$ & 3.95 & 1.22 \\
& $(5)$ & 4.21 & 1.17 \\
\hline d003 & $(1)$ & 5.73 & 2.05 \\
& $(2)$ & 5.67 & 0.89 \\
& $(3)$ & 3.25 & 0.91 \\
& $(4)$ & 5.12 & 1.99 \\
& $(5)$ & 2.66 & 0.66 \\
& $(6)$ & 2.72 & 0.23 \\
\hline
\end{tabular}

\section{Photometry}

Photometric calibration of stacked pawprint images and tile images was performed using numerous detected 2MASS stars. The calibration procedure closely follows that of WFCAM (Hodgkin et al. 2009). Internal photometric accuracy is of the order of $\pm 2 \%$, and for the $J, H$ and $K_{\mathrm{s}}$ bands a similar accuracy as for 2MASS is achieved for most of the survey area. In particularly high-extinction regions and for the $Z$ and $Y$ filters the photometric calibration errors are somewhat larger.

Figure 9 shows the photometric errors as a function of the magnitude computed by the CASU pipeline for the five passbands in a typical disk field (d003) and in an extreme case of a crowded bulge field close to the Galactic Centre (b305). Different extinction $\left(E(B-V)_{\mathrm{d} 003}=1.77, E(B-V)_{\mathrm{b} 305}=\right.$ 1.37 mag; Schlegel et al. 1998) and crowding levels as well as distinct observing strategies between disk and bulge areas (see Sect. 2.3) contribute to the shallower curves seen for the bulge data in all five passbands. The accuracy in the photometry can also be checked using the overlapping regions between the tiles. Figure 10 shows in the top panels the $K_{\mathrm{s}}$ photometry for stellar sources (also computed by the VDFS pipeline at CASU) in the overlapping area between tiles b305 and b291, and between $\mathrm{d} 003$ and $\mathrm{d} 041$. The $5 \sigma$ magnitude limits reached 


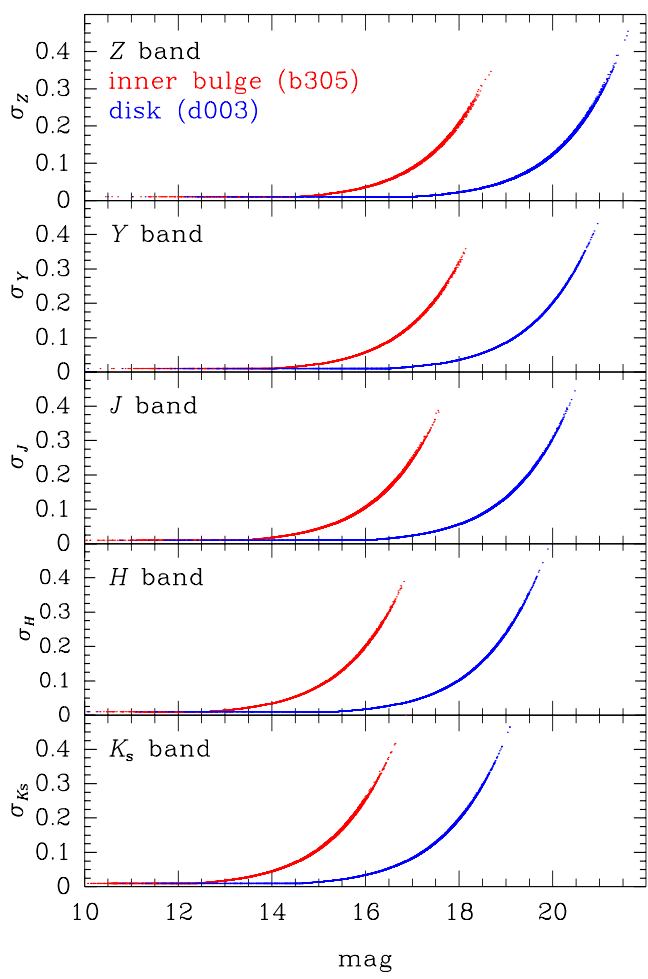

Fig. 9. Photometric error as a function of magnitude for each passband, $Z Y J H K_{\mathrm{s}}$, for a representative field in the disk (d003) and for a crowded bulge field close to the Galactic Centre (b305). We note that different exposure times between disk and bulge areas also contribute to the shallower curve for the bulge data.

for each passband for both bulge and disk tiles as computed by the pipeline are shown in Figs. 3-5.

\subsection{Saturation}

VIRCAM detectors saturate at different levels, mostly around 33 000-35 000 ADU. Detector \#5 has the shallowest well depth (saturation at 24000 ADU), but detector \#13 has the highest non-linearity with of $\sim 10 \%$ at 10000 ADU. This, combined with the fairly bright near-IR sky, results in a restricted dynamic range in the photometry.

Prior to reaching saturation, the VISTA detectors have a nonlinear regime. Although linearity correction is performed in the pipeline data reduction based on observations of an illuminated dome screen, for the stars close to the saturation limit there are still quite significant deviations with respect to 2MASS because of the residual non-linearity. This can be seen clearly in Fig. 2 of Gonzalez et al. (2011a) and also in the VMC survey paper by Cioni et al. (2011).

The overlapping regions also help us to check the linearity of the photometry at the saturation limit. In the top panels of Fig. 10 we see that for both bulge and disk data, several of the brightest stars slightly scatter from the linear distribution close to the saturation level, even taking into account that these are "-1" (stellar) sources in all VDFS single band catalogues from CASU. Some saturated stars are also present in the CASU catalogues (flagged as "-9", see Fig. 7), but these are not present in Fig. 10.

\subsection{Photometric completeness}

Although the main goal of this paper is to describe the content of the first data release for the VVV survey, we now demonstrate briefly how strongly the completeness of the source catalogues provided by CASU depends on the location of the selected area within the survey.

To do this, we carried out artificial star experiments, comparing the detection rate for artificial stars (AS) added to the images using the CASU source detection package imcore (Irwin et al. 2004). We selected two different tiles from the bulge area, namely b204 and b314, which represent different levels of crowding. From these tiles we cut a small $2000 \times 2000$ pixel $^{2}$ area $(11: 3 \times 11: 3)$ for the completeness tests. The two fields were centred on $\alpha_{2000}=18: 12: 13.173, \delta_{2000}=-38: 07: 49.220$ for b204 $\left(l, b=354.72^{\circ},-9.37^{\circ}\right)$, and $\alpha_{2000}=17: 29: 28.920$, $\delta_{2000}=-36: 00: 09.800$ for b314 $\left(l, b=352.21^{\circ},-0.92^{\circ}\right)$, respectively. The field b204 represents a less crowded section of the survey area, whereas b314, close to the Galactic Centre, is one of the most crowded fields. The $K_{\mathrm{s}}$ band source catalogues for the complete tile and the respective sub-sections contain 482, 004/11, 584 (b204) and 1, 137, 615/23, 691 (b314) sources. We point out that in a preliminary test we applied the source detection package to the complete b204 tile and confirmed the number of sources obtained by the CASU pipeline, which validates the source detection in the AS experiments.

In our completeness tests we added 5000 AS to the original images for 16 individual magnitude intervals, each 0.2 mag wide. The positions of these stars were chosen randomly, but the same positions and $K_{\mathrm{s}}$ magnitudes were used for both fields. To create the AS, we derived a single point spread function (PSF), using isolated stars within the corresponding image. We leave the completeness test, which was conducted with a variable PSF for later. The AS images were constructed using the IRAF task addstar ${ }^{9}$.

Using imcore, we created source catalogues for each of the AS images, which we call "output" and compared them to the original source list "input". The ratio of recovered AS to the original 5000 stars for a magnitude range between $12.0 \leq K_{\mathrm{s}} \leq$ $18.2 \mathrm{mag}$ is shown in Fig. 11. In the first test (solid lines) we considered an AS detection to be positive if a source was found within 1 pixel of the inserted position. However, in addition to the simple detection we also need to know with which accuracy the "input" magnitude is recovered. Based on the AS experiments of the "ACS globular cluster survey" (Sarajedini et al. 2007; Anderson et al. 2008) we first allowed a 0.75 mag offset between input and output magnitude, which we finally reduced to $0.5 \mathrm{mag}$. This is based on the assumption that the stellar densities in both our fields are significantly lower than that within the central region of a globular cluster that is targeted by the ACS globular cluster survey. The completeness curve for those tests is shown in Fig. 11 by dashed lines.

Based on the different completeness pattern we find that the source detection efficiency reaches $50 \%$ for stars with $17.8 \lesssim$ $K_{\mathrm{s}} \lesssim 18.1 \mathrm{mag}$ in field b204, and $16.4 \lesssim K_{\mathrm{s}} \lesssim 16.9$ mag in field b314, respectively, depending on the restrictions applied. We also note that the completeness is somewhat lower for the brightest stars (12.0 to $12.2 \mathrm{mag}$ ) in both fields. A possible explanation may be that those stars are close to the saturation limit and the photometric accuracy is therefore reduced.

\section{Comparison with 2MASS photometry}

The VVV observations are approximately four magnitudes deeper than 2MASS. In addition, a very important factor is

\footnotetext{
9 IRAF is distributed by the National Optical Astronomy Observatories, which are operated by the Association of Universities for Research in Astronomy, Inc., under cooperative agreement with the US National Science Foundation.
} 
R. K. Saito et al.: VVV DR1

inner bulge
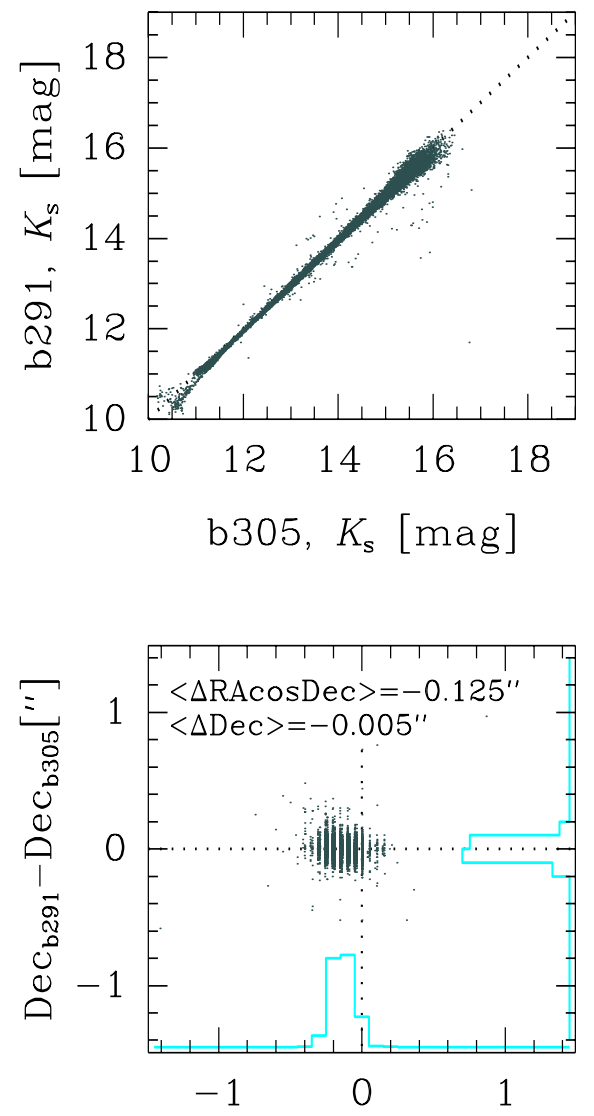

$\left(\mathrm{RA}_{\mathrm{b} 291}-\mathrm{RA}_{\mathrm{b} 305}\right) \cos \mathrm{Dec}["]$ disk
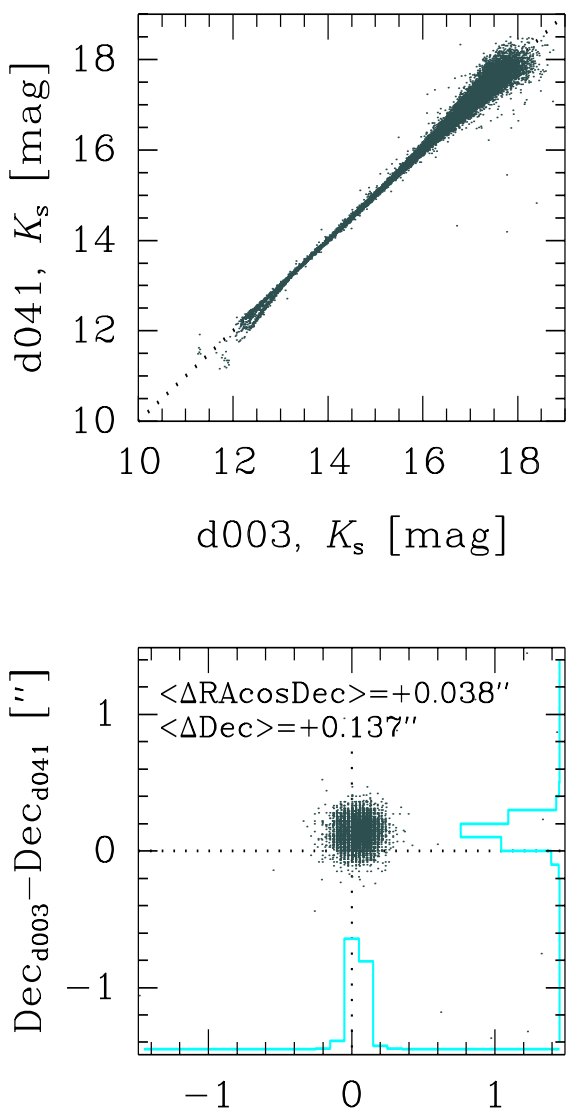

$\left(\mathrm{RA}_{\mathrm{d} 003}-\mathrm{RA}_{\mathrm{d} 041}\right) \cos \mathrm{Dec}["]$
Fig. 10. Top panels: photometry in the $K_{\mathrm{s}}$-band for the overlapping region between tiles b291 and b305 (left), and d003 and d041 (right). Only stellar sources were used in these plots. Bottom panels: astrometric accuracy for the same overlapping regions. The mean values for $\Delta \alpha \times \cos \delta$ and $\Delta \delta$ are shown in the top left corner. Counting histograms for the distribution are also shown for both axes.

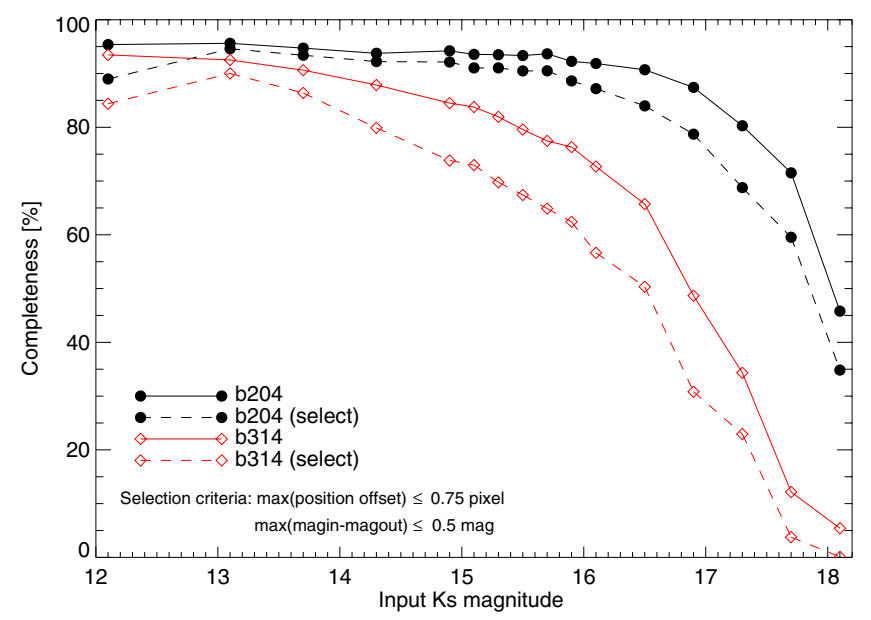

Fig. 11. $K_{\mathrm{s}}$-band completeness test for two $2000 \times 2000$ pixels $^{2}(11$ ' $3 \times$ $11: 3$ ) fields from the bulge area of the VVV survey. The fields were selected to represent a less crowded region (tile b204) as well as one of the most crowded regions near the Galactic Centre (tile b314). Solid points correspond to b204, whereas open diamonds represent b314. In addition we applied selection criteria to define a confirmed source detection in our AS experiments (see text for details). Solid lines show the completeness fraction if a source was detected within a radius $\leq 1$ pixel of its original position. Reducing this radius to 0.5 pixel and requiring the original $K_{\mathrm{s}}$-band magnitude to be recovered within 0.5 mag results in the completeness curves shown with dashed lines.

the excellent image quality (with seeing $00^{\prime} 9-1^{\prime \prime} .0$ ) in the entire VVV survey area for the multi-band, single epoch observations.
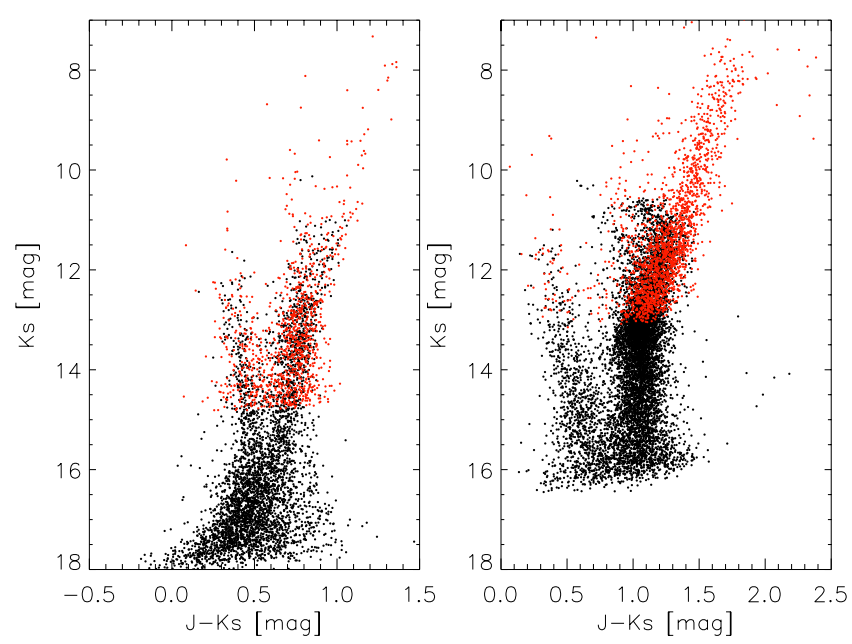

Fig. 12. CMDs comparing VVV (black) and 2MASS data (red) for the bulge area. The left-hand panel shows a field in the outer bulge (b235) while the right-hand panel shows one of the most crowded bulge fields (b305), close to the Galactic Centre.

This allows us to reach the red clump magnitude across the entire bulge (Gonzalez et al. 2011a; Saito et al. 2011), and therefore to study the stellar populations and the structure of the inner Galaxy to an unprecedented level of detail, such as the identification of RR Lyrae and derivation of accurate distances. Figure 12 shows a CMD for $10^{\prime} \times 10^{\prime}$ regions in the inner bulge (b305) and the outer bulge (b235) of VVV stellar sources compared to 
A\&A 537, A107 (2012)
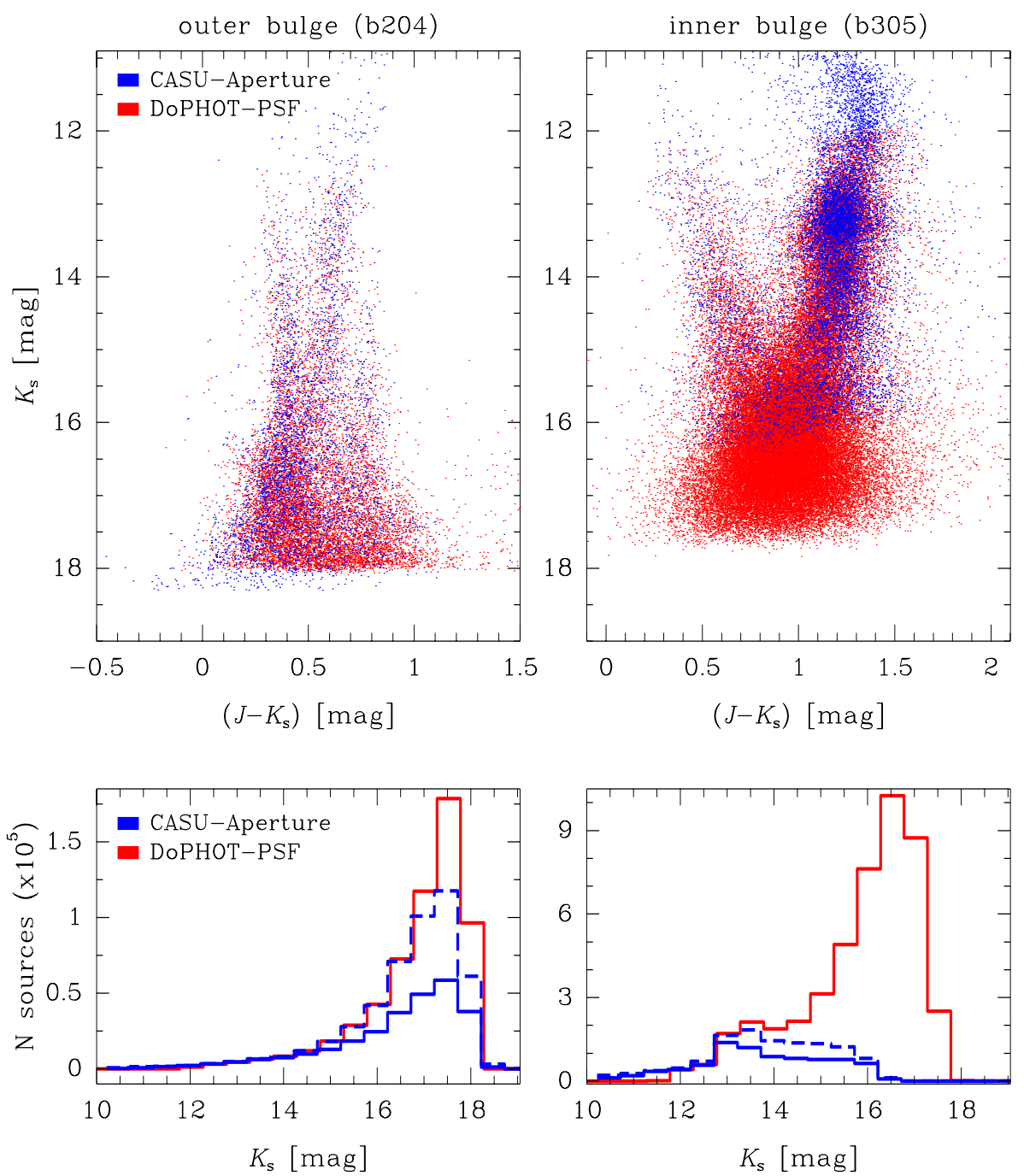

Fig. 13. Top left panel: $K_{\mathrm{s}}$ vs. $\left(J-K_{\mathrm{s}}\right)$ CMD for a less crowded bulge field (a section of tile b204) comparing the $5 \sigma$ magnitude limit of the CASU aperture photometry with PSF photometry (also with $5 \sigma$ magnitude limit). The bottom left panel shows histograms for all sources (dashed line) and only the stellar objects (solid line) found in tile b204, also in comparison with the PSF photometry. The right-hand panels show the same analysis for a highly crowded field close to the Galactic Centre (tile b305).
2MASS CMDs of the same regions. Because the VVV photometry is much deeper, it allows us not only to trace the red clump even in the most extincted regions, but also to study the stellar populations behind the bulge. A detailed study of the colour transformations between VISTA and 2MASS photometric systems for VVV disk fields will be presented by Soto et al. (in prep.).

\section{VVV DR1 catalogues vs. PSF photometry}

The photometric catalogues published in this, as well as any following VVV data release, are based on aperture photometry only, computed by the CASU pipeline on individual tiles (see Sect. 2.4). However, for very crowded fields (e.g., the innermost Galactic Centre or the central region of stellar clusters), more complete and deeper photometry can be obtained with PSF photometry. In this section we present tests performed by VVV team members using PSF photometry on the VVV images. The results show that the PSF fitting can reach up to 1.5 mag deeper than aperture photometry, detecting up to twice more sources for highly crowded fields, where aperture photometry is known to be inefficient, particularly for faint sources. We emphasize that the PSF data are not part of the VVV DR1.

The tests were performed using the apermag 3 aperture fluxes, which are used in the CASU catalogues as well as in the VSA database as the default values to represent the flux for all images. However, the CASU pipeline measures positions and fluxes for different concentric apertures designed to adequately sample the curve-of-growth of the majority of images. The apermagl has 1" diameter and each successive aperture increases by a factor of $\sqrt{2}$ in diameter. For highly crowded fields the aperture photometry using the apermag 3 can simultaneously fit multiple overlapping sources, but it does not perform any subtraction in order to check for fainter sources hidden underneath. In this case the apermag 1 and apermag 2 can be more suitable than apermag3, even taking into account that narrow apertures have more uncertain aperture corrections, especially in poor seeing conditions.

Figure 13 shows in the top-left panel the $K_{\mathrm{s}}$ vs. $\left(J-K_{\mathrm{s}}\right) \mathrm{CMD}$ for a field with relatively low crowding and extinction (a section of tile b204), comparing aperture photometry performed by CASU with PSF photometry obtained with DoPHOT (Schechter et al. 1993) for the same field, using $5 \sigma$ magnitude limits in both cases. All structures are correctly seen in both cases, and both aperture and PSF photometries reach $K_{\mathrm{s}} \sim 18.0 \mathrm{mag}$.

The bottom-left panel of Fig. 13 shows the magnitude distribution of all sources found in the CASU catalogue (dashed line) as well as that of stellar sources only (solid line). Even taking into account that aperture photometry contains some brighter stars that are not present in the PSF photometry, the total number of sources found by PSF photometry in the $K_{\mathrm{s}}$-band image of b204 is 589187 , in comparison to 482004 (all sources) and 273550 (stellar flag) sources present in the CASU catalogue. 

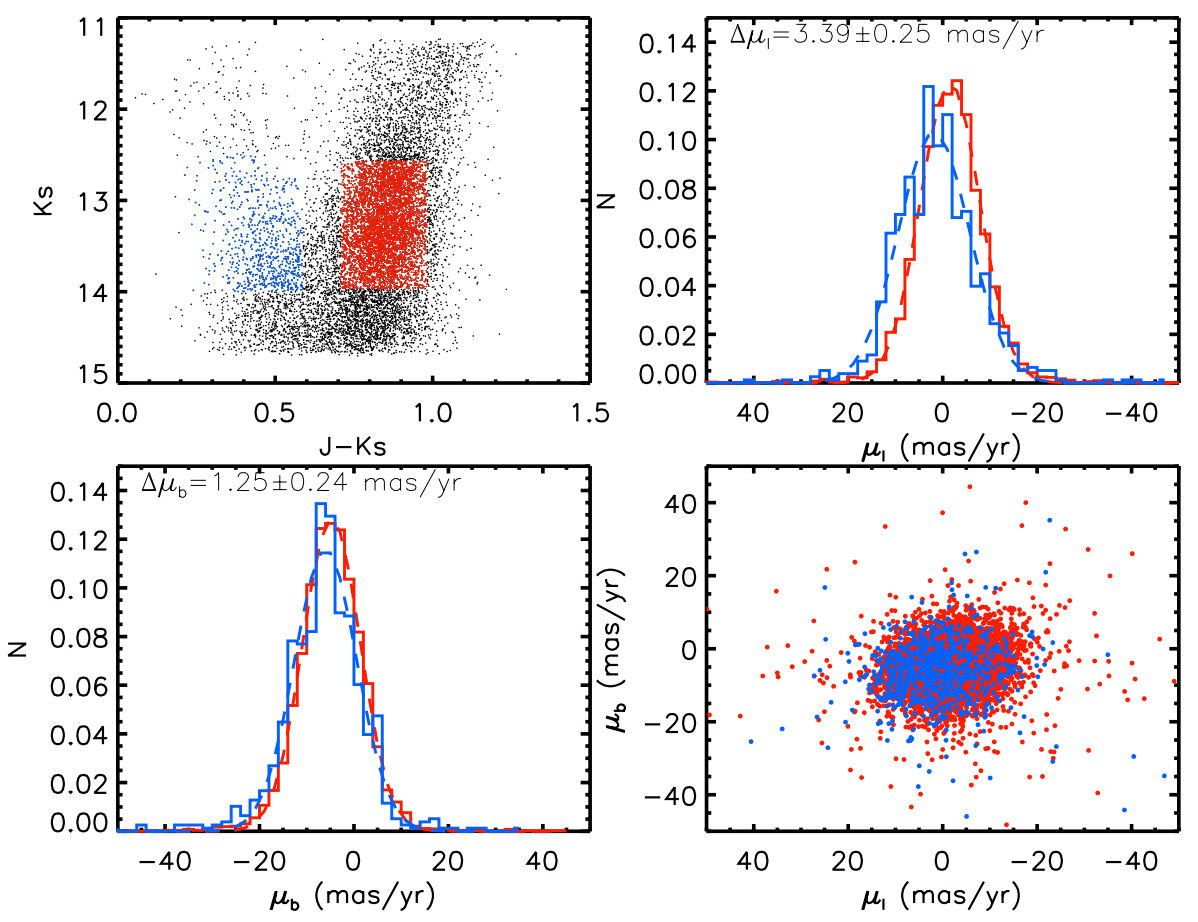

Fig. 14. Top left panel: $K_{\mathrm{s}}$ vs. $\left(J-K_{\mathrm{s}}\right)$ CMD for a $10 \times 10 \mathrm{arcmin}^{2}$ region in tile b249 for VVV stellar sources matched with 2MASS. The CMD is centred in the region of the bulge red clump (red) and the disk main sequence (blue). Top right panel: histogram of the longitudinal proper motion for both selected regions in the CMD. The mean values for $\Delta \alpha \times \cos \delta$ and $\Delta \delta$ are shown in the top left corner. Bottom left panel: same as top right panel for the latitudinal proper motion distribution. Bottom right panel: the $\mu_{b}$ vs. $\mu_{l}$ distribution in units of mas $\mathrm{yr}^{-1}$.
For comparison, the same analysis is performed in the right panel of Fig. 13 for the inner bulge field b305, where the effects of crowding and extinction are significant. The PSF photometry allows us not only to resolve the high-density areas, but it also reaches $\sim 1.5$ mag deeper, i.e., $K_{\mathrm{s}} \sim 17.5 \mathrm{mag}$, while the aperture photometry is limited to $K_{\mathrm{s}} \sim 16.0 \mathrm{mag}$. The bottom-right panel of Fig. 13 shows by how much the PSF photometry exceeds the aperture photometry for faint sources. The total number of sources found by PSF photometry for field b305 in the $K_{\mathrm{s}}$-band is 4601529 in comparison to 1201557 , (all flags) and 818706 (stellar only) sources present in the CASU catalogue.

Tests performed by VVV team members show that the PSF photometry can be even deeper using a DAOPHOTALLFRAME suite of routines (Stetson 1994) customized for the VVV data (Mauro et al., in prep.).

\section{Astrometry}

The native VISTA WCS distortion model for pawprints is based on zenith-pole-north (ZPN) projection and is available in image headers. The distortions are radial and are well described by

$r^{\prime}=k_{1} \times r+k_{3} \times r^{3}+k_{5} \times r^{5}$,

with $k_{1}=0.3413 \mathrm{arcsec} / \mathrm{pix}$ being the plate scale at the centre, and $k_{3} / k_{1}=44, k_{5} / k_{1}=10300$ are distortion coefficients in angular units of radians. Higher order terms are negligible.

The median WCS rms is $\sim 70$ mas and is dominated by the 2MASS astrometric errors. We note that in the coadded pawprints as well as in the complete tiles the pixels were resampled to a common spatial scale and the astrometric distortions were removed according to Eq. (1). The resampling was also required in the pawprints because the jitter offsets are sufficiently large (i.e., 15'.0) to affect the coadding (see also Table 3) owing to the size of the distortions. Another way to evaluate the internal astrometric accuracy is to use overlaps between different tiles. Hundreds of stars are detected independently in two adjacent tiles. Figure 10 shows in the top panels the photometry in $K_{\mathrm{s}}$ for overlapping regions between tiles in the inner bulge (b291 and b305) and disk (d003 and d041) areas. Different observing strategies, in particular exposure times (see Table 3), for the disk and bulge areas, as well as the high background brightness owing to unresolved stars in the inner bulge, make the bulge photometry shallower than that in the disk region. The astrometric accuracy is shown in the bottom panels of Fig. 10 in terms of the distribution $\Delta \delta$ vs. $\Delta \alpha \times \cos \delta$. Typical values for the astrometric accuracy are $\sim 25$ mas for a $K_{\mathrm{s}}=15.0$ mag source and $\sim 175$ mas for $K_{\mathrm{s}}=18.0 \mathrm{mag}$. Despite the shorter time baseline of the VVV survey, the typical proper motion measurements should reach the accuracy between $\sim 7 \mathrm{mas} \mathrm{yr}^{-1}\left(K_{\mathrm{s}}=15.0 \mathrm{mag}\right)$

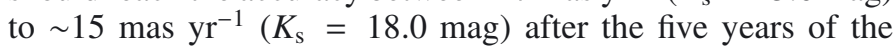
VVV campaign.

A test for these measurements can already be achieved using 2MASS and VVV datasets, which provide a time baseline of 11 years. Cross-matching of sources between VVV and 2MASS was performed for a $10 \times 10 \mathrm{arcmin}^{2}$ field in tile b249. The astrometric differences between stellar sources in these two catalogues can be used to derive proper motions of stars in terms of $\mu_{l}$ and $\mu_{b}$. Clarkson et al. (2008), based on two epochs of HST imaging, showed that foreground disk stars observed in the colour-magnitude diagrams towards the bulge can be separated based on Gaussian fits to the distribution of their proper motions with mean differences of $\left(\Delta \mu_{l}, \Delta \mu_{b}\right)=(3.22 \pm 0.15,0.81 \pm$ 0.13) mas $_{\mathrm{yr}^{-1}}$.

Figure 14 shows the selection of stars in the CMD for matched stars between VVV and 2MASS that belong to disk and bulge populations together with the distribution of $\mu_{l}$ and $\mu_{b}$ for each selection. Although the distributions are wider than those presented in the HST analysis of Clarkson et al. (2008), the mean differences between proper motions of disk and bulge are still evident based on these data showing differences of $\left(\Delta \mu_{l}, \Delta \mu_{b}\right)=(3.39 \pm 0.25,1.25 \pm 0.24) \mathrm{mas} \mathrm{yr}^{-1}$, in good agreement with those of Clarkson et al. (2008).

\section{VVV Source maps}

Figures 15 and 16 show all objects with stellar flag detected in each tile in the DR1 data. Only stars matched in the $J, H$ and $K_{\mathrm{s}}$ filters have been plotted. The total number of sources 


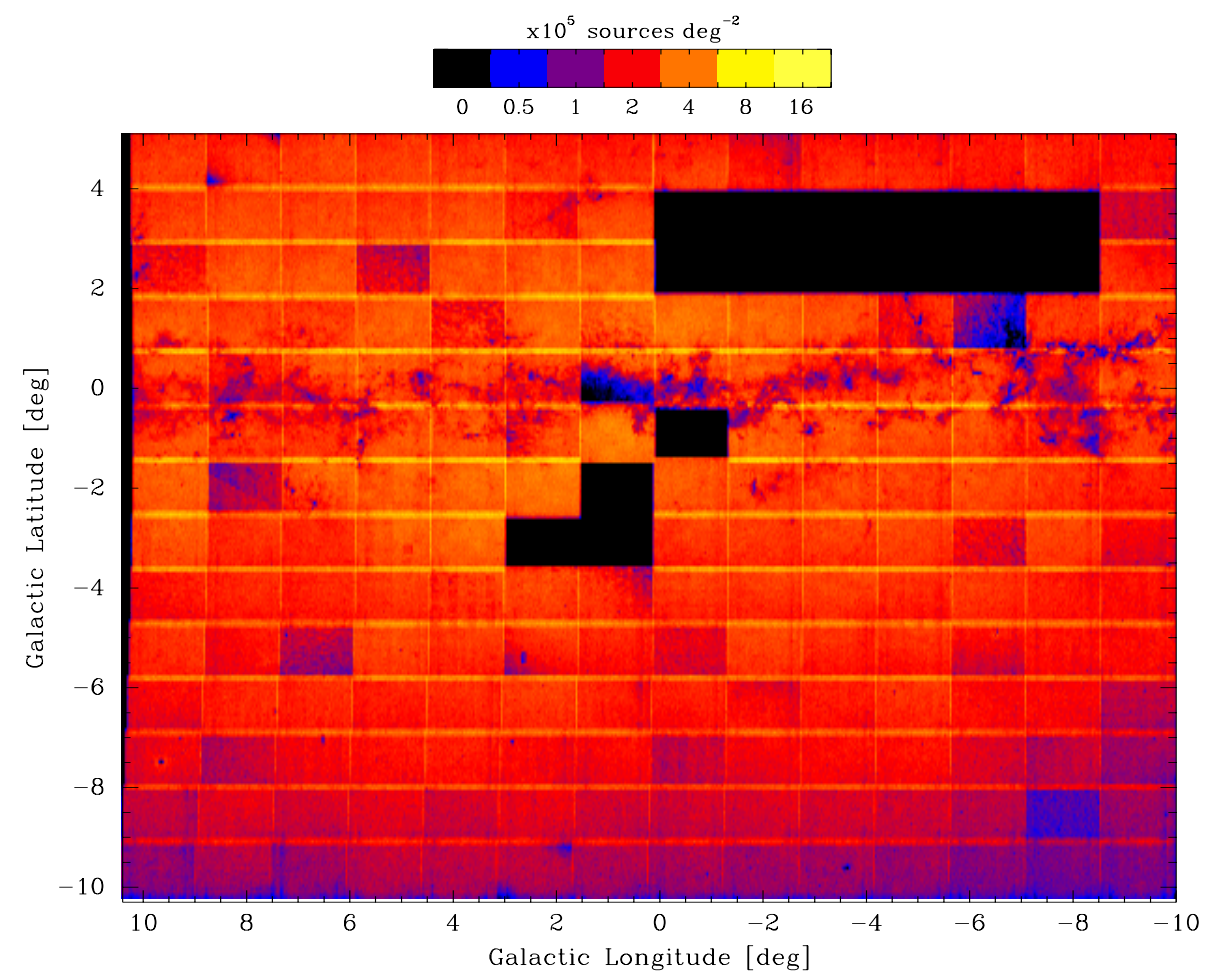

Fig. 15. Density map in logarithmic scale showing the VVV bulge area. The map was made using all stellar point sources detected in the $J, H$, and $K_{\mathrm{s}} 1.1 \mathrm{CASU}$ catalogues. Crowded areas appear in yellow, while less populated regions and high-extinction areas are shown in blue. The overlapping regions between the tiles are highlighted because the point sources are detected twice, which generates the grid pattern that also indicates the size of overlap regions. The density, in units of $10^{5}$ sources $\mathrm{deg}^{-2}$, is indicated in the horizontal bar at the top.

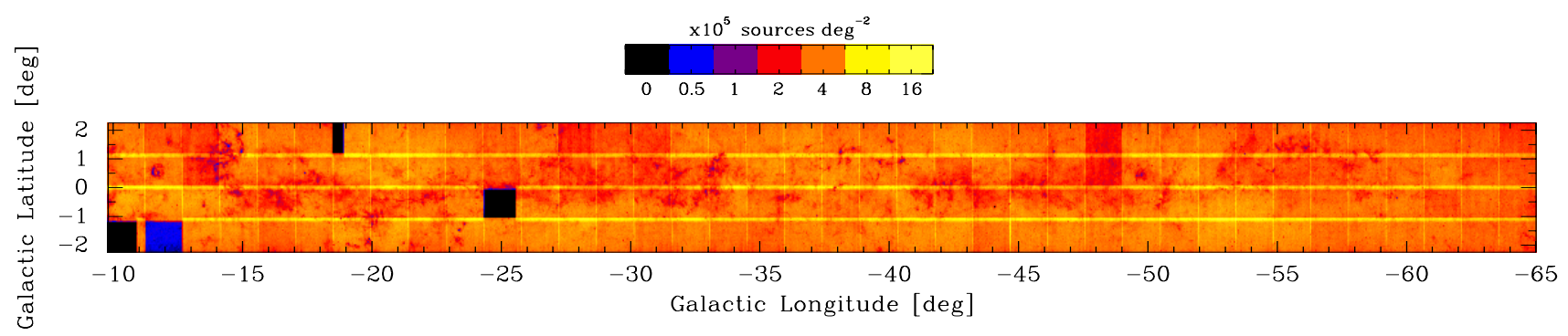

Fig. 16. Density map in logarithmic scale showing the VVV disk area. The notation is similar to that presented in Fig. 15.

found in the bulge region is $7.06 \times 10^{7}$, while the disk has $9.29 \times 10^{7}$ sources. The stellar density (corrected for the total field size) is higher in the disk because of the deeper photometry in all five filters compared to the bulge observations (see Table 3, and Figs. 3-5).

Note that these density maps already provide a wealth of information on the extinction and the structure of the inner Galaxy, which will be investigated in detail in subsequent papers (e.g., Gonzalez et al. 2011a,b; Saito et al., in prep.).

The regular grid pattern seen in both the bulge and disk areas is due to the overlap of adjacent tiles. The individual catalogues used in Figs. 15 and 16 contain $\sim 10 \%$ of the total number of sources twice, contributed by two independent tiles, and leading to the much higher source density shown in the two figures. The detection of a significant percentage of sources in two independent tiles not only helps to test and/or confirm the photometric and astrometric calibration, but also to carry out quality control. These density maps are also used to identify problematic tiles, i.e., incomplete data readouts, missing tiles, and observations made under strongly varying seeing conditions (e.g., tiles b216 and b343).

Moreover, those overlap regions will also be beneficial for one of the main scientific goals of the VVV survey. Because the different tiles are observed independently (with the exception of concatenated tiles), the variable stars included on those tiles will obtain twice as many $K_{\mathrm{s}}$ epochs, and hence much better sampled light curves (see also Catelan et al. 2011).

\section{Difference image testing}

Whilst DR1 does not include photometry based on difference image analysis (DIA), we have tested the suitability of the data for DIA analysis based on a modified double-pass version of the ISIS package Alard \& Lupton (1998) originally developed for the Angstrom M31 microlensing survey (Kerins et al. 2010).

Currently, the VVV science verification (SV) field comprises the largest number of epochs and therefore most DIA testing has been performed on this dataset. One problem with difference imaging of VVV data is that often the seeing is so good that the point spread function (PSF) is poorly sampled by the pixel size of around 0.34 . For good DIA kernel convolution we usually require more than 2.5 pixels/FWHM. To minimize undersampling, instead of convolving a good seeing reference image to a poorer seeing target, we selected a poor seeing reference frame and convolved the target image to it. The DIA image is therefore constructed by minimizing

$D_{i, j}^{2}=\min \sum_{i, j}\left[R_{i, j}-(T \otimes K)_{i, j}+B_{i, j}\right]^{2}$ 

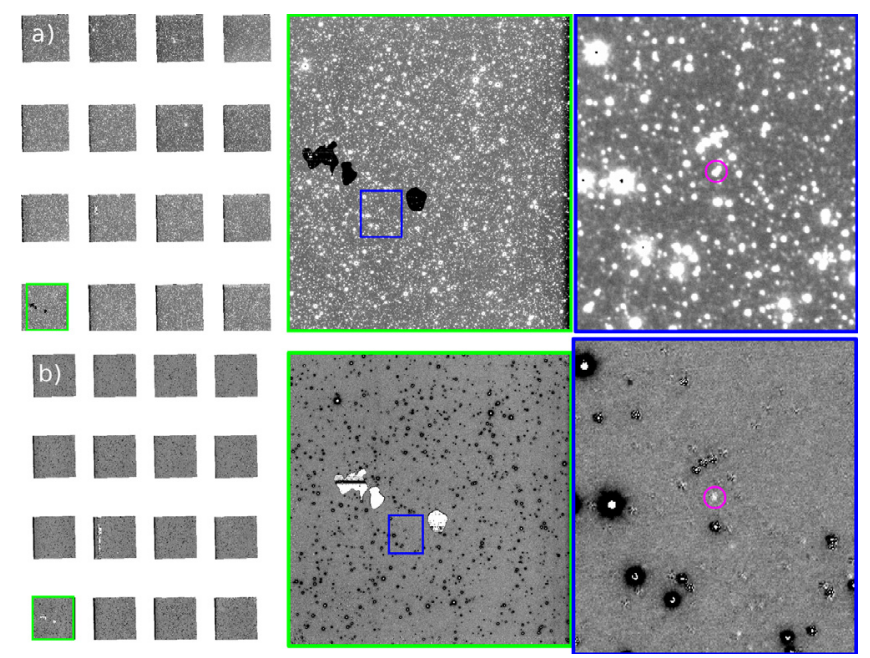

Fig. 17. a) $K_{\mathrm{s}}$ band pawprint from one VVV SV bulge field epoch showing views of the full pawprint (left); a zoom into array 1 (middle); and another zoom centred on a circled variable object (right). b) The bottom row shows the respective difference image views. Blackened objects are DIA residuals around saturated stars, which comprise a non-negligible fraction of the image area for bulge fields.

where the sum is over the pixel coordinates $(i, j), R$ and $T$ are the reference and target images, $B$ describes the differential background between $R$ and $T, K$ is the convolution kernel and $D$ is the resulting difference image (see Alard \& Lupton 1998, for details of how $K$ is constructed). Before difference imaging we added the sky images back onto the sky-subtracted image stacks.

Figure 17 shows a $K_{\mathrm{S}}$ band pawprint from one epoch of the VVV SV bulge field. The lower row shows the resulting difference images. Whilst the difference image quality is reasonably clean, there are many black residuals occurring around the many saturated stars in the field. However, variable objects are as easy to pick out as in the circled example in the zoomed DIA panel in Fig. 17.

The background noise level of the DIA images is consistent across all arrays and performs well with respect to imcore sky noise estimates (Irwin et al. 2004). In Fig. 18 we plot the pixel histograms of DIA flux for the same SV pawprint shown in Fig. 17 for each of the 16 arrays. The fluxes are normalised to a level that is just $40 \%$ of the sky noise estimate reported by imcore. In all cases the histograms show that difference imaging noise is significantly below that predicted by imcore, as shown by the close resemblance of the normalised DIA flux distributions to a unit Gaussian curve.

The photometric performance of VVV DIA will be calibrated in greater detail in a future paper.

\section{Summary}

We have presented the VVV Data Release 1, describing the design, observations, data processing, data quality, and limitations. The data are of very high quality, and represent a vast improvement over existing near-IR photometry. Therefore they are useful for a wide variety of studies such as open/embedded clusters (Borissova et al. 2011; Baume et al. 2011), globular clusters (Minniti et al. 2011a; Moni Bidin et al. 2011), distance scale (Majaess et al. 2011), YSO censuses (Faimali et al., in prep.), brown dwarfs (Folkes et al., in prep.), proper motions (Minniti et al., in prep.), disk stellar populations and variable stars (Pietrukowicz et al. 2012), Galactic structure of the disk

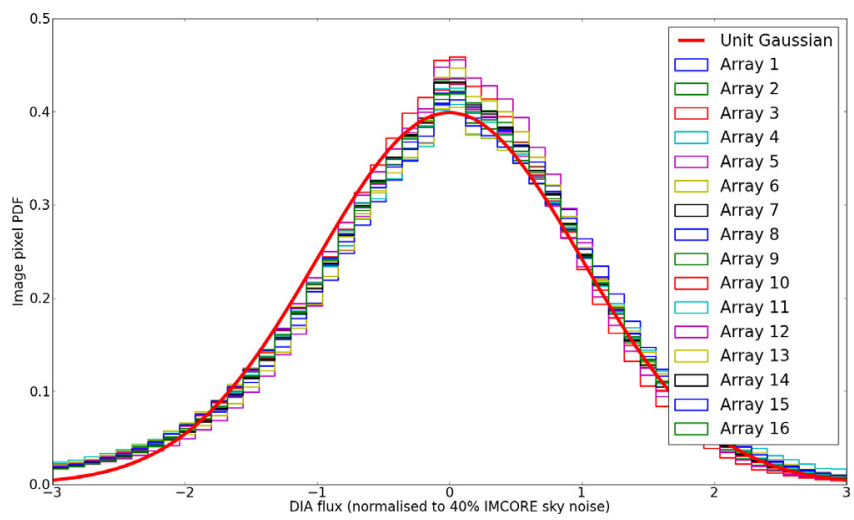

Fig. 18. Noise histograms for the $K_{\mathrm{s}}$ band DIA images shown in row b) of Fig. 17. The $x$-axis is difference-flux normalised to $40 \%$ of the sky noise level reported by imcore. The smooth function is a unit Gaussian, which indicates that for this image the DIA noise is reasonably Gaussian for all arrays and is characterised by a noise level that is well below the imcore sky noise estimate.

mapping the edge of the stellar disk (Minniti et al. 2011b), and the inner structure of the bulge (Gonzalez et al. 2011b), bulge stellar populations including metallicity, extinction and dust maps (Gonzalez et al. 2011a; Catelan et al., in prep.) ${ }^{10}$ highenergy sources (Greiss et al. 2011a,b; Masetti et al., in prep.), background galaxies (Amôres et al., in prep.), colour transformations between VISTA and 2MASS systems (Soto et al., in prep.), as well as enabling and complementing other Galactic structure and stellar population studies, variable star studies in clusters and in the field (pulsating variables, eclipsing binaries, and planetary transits), gravitational microlensing studies, Galactic Centre studies, ultra-high-velocity star searches, PNe searches, SN light echo searches, QSO searches, searches for faint solar system objects (e.g., NEOs, MBAs, LJ5s, TNOs), etc.

Acknowledgements. We gratefully acknowledge use of data from the ESO Public Survey programme ID 179.B-2002 taken with the VISTA telescope, data products from the Cambridge Astronomical Survey Unit, and funding from the FONDAP Center for Astrophysics 15010003, the BASAL CATA Center for Astrophysics and Associated Technologies PFB-06, the FONDECYT from CONICYT, and the Ministry for the Economy, Development, and Tourism's Programa Iniciativa Científica Milenio through grant P07-021-F, awarded to The Milky Way Millennium Nucleus. R.K.S. and D.M. acknowledge financial support from CONICYT through Gemini Project No. 32080016. D.M. acknowledge support by Proyecto FONDECYT Regular No. 1090213. M.Z. and O.A.G. acknowledge support by Proyecto FONDECYT Regular No. 1110393. J.B. and F.P. are supported by FONDECYT Regular No. 1080086. J.R.A.C. is supported by CONICYT through Gemini Project No.Ãl'32090002. A.N.C. received support from Comitee Mixto ESO-Gobierno de Chile. M.S. acknowledges support by Proyecto FONDECYT Regular No. 3110188 and Comite Mixto ESOGobierno de Chile. Support for R.A. is provided by Proyecto FONDECYT Regular No. 3100029. R.d.G. acknowledges partial research support through grant 11073001 from the National Natural Science Foundation of China. M.M.H. is supported for this work by the US National Science Foundation under Grant Nos. 0607497 and 1009550. G.P. acknowledge support from the Millennium Center for Supernova Science through grant P06-045-F funded by Programa Bicentenario de Ciencia y Tecnologa de CONICYT. M.C., J.A.G. and I.D. acknowledge support by Proyecto FONDECYT Regular No. 1110326. M.C. is also supported in part by Proyecto Anillo ACT-86. S.L.F. acknowledges funding support from the ESO-Government of Chile Mixed Committee 2009 and from the Gemini-CONICYT grant No. 32090014/2009. B.B., B.D. and L.S.J. acknowledge support from FAPESP and CNPq. E.B.A. thanks Fundação para a Ciência e Tecnologia (FCT) under the grant SFRH/BPD/42239/2007. R.K. acknowledges support from the Centro de Astrofisica de Valparaiso and Proyecto DIUV23/2009.

10 The Bulge Extinction and Metallicity calculator based on VVV maps is available at:

http://www. eso.org/ ogonzale/BEAMEC/calculator.php 


\section{References}

Alard, C., \& Lupton, R. 1998, ApJ, 503, 325

Alcock, C., Allsman, R. A., Alves, D. R., et al. 2000, ApJ, 541, 734

Amôres, E. B., \& Lépine, J. R. D. 2005, AJ, 130, 659

Anderson, J., Sarajedini, A., Bedin, L. R., et al. 2008, AJ, 135, 2055

Arnaboldi, M., Neeser, M. J., Parker, L. C., et al. 2007, The Messenger, 127, 28

Baume, G., Carraro, G., Comeron, F., \& de Elía, G. C. 2011, A\&A, 531, A73

Benjamin, R. A., Churchwell, E., Babler, B. L., et al. 2003, PASP, 115, 953

Borissova, J., Bonatto, C., Kurtev, R., et al. 2011, A\&A, 532, A131

Cardelli, J. A., Clayton, G. C., \& Mathis, J. S. 1989, ApJ, 345, 245

Catelan, M., Minniti, D., Lucas, P. W., et al. 2011, RR Lyrae Stars, Metal-Poor Stars, and the Galaxy, 145

Cioni, M.-R. L., Clementini, G., Girardi, L., et al. 2011, A\&A, 527, A116

Clarkson, W., Sahu, K., Anderson, J., et al. 2008, ApJ, 684, 1110

Cutri, R. M., Skrutskie, M. F., van Dyk, S., et al. 2003, The IRSA 2MASS AllSky Point Source Catalog, NASA/IPAC Infrared Science Archive

Dalton, G. B., Caldwell, M., Ward, A. K., et al. 2006, Proc. SPIE, 6269

Dobashi, K., Uehara, H., Kandori, R., et al. 2005, PASJ, 57, 1

Drimmel, R., Cabrera-Lavers, A., \& López-Corredoira, M. 2003, A\&A, 409, 205

Emerson, J. P., \& Sutherland, W. J. 2010, Proc. SPIE, 7733

Epchtein, N., de Batz, B., Copet, E., et al. 1994, Ap\&SS, 217, 3

Froebrich, D., Ray, T. P., Murphy, G. C., \& Scholz, A. 2005, A\&A, 432, L67

Gonzalez, O. A., Rejkuba, M., Zoccali, M., Valenti, E., \& Minniti, D. 2011a, A\&A, 534, A3

Gonzalez, O. A., Rejkuba, M., Minniti, D., et al. 2011b, A\&A, 534, L14

Greiss, S., Steeghs, D., Maccarone, T. J., et al. 2011a, Astronomer's Telegram, 3562

Greiss, S., Steeghs, D., Maccarone, T. J., et al. 2011b, Astronomer's Telegram, 3695

Hilker, M., Primas, F., \& Comerón, F. 2011, Survey Area Definition Tool Cookbook for VISTA

Hodgkin, S. T., Irwin, M. J., Hewett, P. C., \& Warren, S. J. 2009, MNRAS, 394, 675

Irwin, M. J., Lewis, J., Hodgkin, S., et al. 2004, Proc. SPIE, 5493, 411

Ivanov, V. D., \& Szeifert, T. 2009, VIRCAM/VISTA User Manual

Kerins, E., Darnley, M. J., Duke, J. P., et al. 2010, MNRAS, 409, 247

Lewis, J. R., Irwin, M., \& Bunclark, P. 2010, Astronomical Data Analysis Software and Systems XIX, 434, 91

Lucas, P. W., Hoare, M. G., Longmore, A., et al. 2008, MNRAS, 391, 136

Majaess, D., Turner, D., Moni Bidin, C., et al. 2011, ApJ, 741, L27
Marshall, D. J., Robin, A. C., Reylé, C., Schultheis, M., \& Picaud, S. 2006, A\&A, 453, 635

Minniti, D., Lucas, P. W., Emerson, J. P., et al. 2010, New A Stron., 15, 433

Minniti, D., Hempel, M., Toledo, I., et al. 2011a, A\&A, 527, A81

Minniti, D., Saito, R. K., Alonso-García, J., Lucas, P. W., \& Hempel, M. 2011b, ApJ, 733, L43

Moni Bidin, C., Mauro, F., Geisler, D., et al. 2011 [arXiv: 1109. 1854]

Nagayama, T., Nagashima, C., Nakajima, Y., et al. 2003, Proc. SPIE, 4841, 459

Nishiyama, S., Nagata, T., Sato, S., et al. 2006, ApJ, 647, 1093

Nishiyama, S., Tamura, M., Hatano, H., et al. 2009, ApJ, 696, 1407

Pietrukowicz, P., Minniti, D., Alonso-Garcia, J., \& Hempel, M. 2012, A\&A, in press, DOI: 10.1051/0004-6361/201116877

Saito, R., Hempel, M., Alonso-García, J., et al. 2010, The Messenger, 141, 24

Saito, R. K., Zoccali, M., McWilliam, A., et al. 2011, AJ, 142, 76

Sarajedini, A., Bedin, L. R., Chaboyer, B., et al. 2007, AJ, 133, 1658

Schechter, P. L., Mateo, M., \& Saha, A. 1993, PASP, 105, 1342

Schlegel, D. J., Finkbeiner, D. P., \& Davis, M. 1998, ApJ, 500, 525

Stetson, P. B. 1994, PASP, 106, 250

Szymański, M. K., Udalski, A., Soszyński, I., et al. 2011, Acta Astron., 61, 83

Taylor, M. B. 2006, Astronomical Data Analysis Software and Systems XV, 351, 666

Udalski, A., Szymanski, M., Kaluzny, J., Kubiak, M., \& Mateo, M. 1993, Acta Astron., 43, 69

\section{Appendix A: VVV tile coordinates}

Here we list the tile centre coordinates for all VVV pointings. There are 196 bulge tiles, with names starting with "b", and 152 tiles in the disk area, whose names start with "d". For each tile we provide tile centre coordinates in Equatorial and Galactic coordinates. All tiles have been observed using an identical offsetting strategy, combining six pawprints to contiguously fill $1.5 \times 1.1$ sq. deg area. The second-last column lists the filters for which the tile has been completed (i.e., observed within constraints), and the last column lists the number of epochs taken in the $K_{\mathrm{s}}$ band within the first observing season (DR1). 
Table A.1. VVV tile centres.

\begin{tabular}{|c|c|c|c|c|c|c|}
\hline $\begin{array}{l}\text { Tile } \\
\text { name }\end{array}$ & $\begin{array}{l}\text { RA (J2000.0) } \\
\text { dd:mm:ss.sss }\end{array}$ & $\begin{array}{c}\text { Dec (J2000.0) } \\
\text { dd:mm:ss.ss }\end{array}$ & $\begin{array}{c}\begin{array}{c}\text { Longitude } \\
\text { degrees }\end{array} \\
\text { deres }\end{array}$ & $\begin{array}{l}\text { Latitude } \\
\text { degrees }\end{array}$ & $\begin{array}{c}\text { Filters } \\
\text { completed }\end{array}$ & $\begin{array}{l}K_{\mathrm{s}} \text { epochs } \\
\text { completed }\end{array}$ \\
\hline $\mathrm{d} 001$ & $11: 43: 24.936$ & $-63: 31: 38.64$ & 295.43770 & -1.64975 & $Z Y J H K_{\mathrm{s}}$ & 5 \\
\hline $\mathrm{d} 002$ & $11: 56: 12.576$ & $-63: 52: 21.00$ & 296.89672 & -1.64979 & $Z Y J H K_{\mathrm{s}}$ & 5 \\
\hline $\mathrm{d} 003$ & 12:09:17.184 & $-64: 08: 46.68$ & 298.35572 & -1.64971 & $Z Y J H K_{\mathrm{s}}$ & 5 \\
\hline $\mathrm{d} 004$ & $12: 22: 35.184$ & $-64: 20: 48.12$ & 299.81470 & -1.64971 & $Z Y J H K_{\mathrm{s}}$ & 5 \\
\hline $\mathrm{d} 005$ & $12: 36: 02.640$ & $-64: 28: 18.84$ & 301.27373 & -1.64973 & $Z Y J H K_{\mathrm{s}}$ & 5 \\
\hline d006 & $12: 49: 35.184$ & $-64: 31: 14.88$ & 302.73271 & -1.64977 & $Z Y J H K_{\mathrm{s}}$ & 5 \\
\hline $\mathrm{d} 007$ & 13:03:08.352 & $-64: 29: 34.44$ & 304.19170 & -1.64978 & $Z Y J H K_{\mathrm{s}}$ & 5 \\
\hline $\mathrm{d} 008$ & $13: 16: 37.632$ & $-64: 23: 18.24$ & 305.65072 & -1.64970 & $Z Y J H K_{\mathrm{s}}$ & 5 \\
\hline d009 & $13: 29: 58.632$ & $-64: 12: 30.24$ & 307.10972 & -1.64971 & $J H K_{\mathrm{s}}$ & 5 \\
\hline $\mathrm{d} 010$ & $13: 43: 07.272$ & $-63: 57: 15.84$ & 308.56873 & -1.64973 & $Z Y J H K_{\mathrm{s}}$ & 5 \\
\hline $\mathrm{d} 011$ & $13: 55: 59.856$ & $-63: 37: 42.60$ & 310.02772 & -1.64971 & $Z Y J H K_{\mathrm{s}}$ & 5 \\
\hline $\mathrm{d} 012$ & 14:08:33.240 & $-63: 14: 00.24$ & 311.48673 & -1.64970 & $Z Y J H K_{\mathrm{s}}$ & 5 \\
\hline $\mathrm{d} 013$ & $14: 20: 44.808$ & $-62: 46: 19.92$ & 312.94573 & -1.64979 & $Z Y J H K_{\mathrm{s}}$ & 5 \\
\hline $\mathrm{d} 014$ & $14: 32: 32.496$ & $-62: 14: 52.80$ & 314.40472 & -1.64974 & $Z Y J H K_{\mathrm{s}}$ & 5 \\
\hline $\mathrm{d} 015$ & $14: 43: 42.144$ & $-61: 40: 33.96$ & 315.83598 & -1.64972 & $Z Y J H K_{\mathrm{s}}$ & 4 \\
\hline d016 & $14: 54: 38.784$ & $-61: 02: 16.44$ & 317.29497 & -1.64975 & $Z Y J H K_{\mathrm{s}}$ & 4 \\
\hline $\mathrm{d} 017$ & 15:05:08.712 & $-60: 20: 51.00$ & 318.75395 & -1.64975 & $Z Y J H K_{\mathrm{s}}$ & 4 \\
\hline $\mathrm{d} 018$ & $15: 15: 11.880$ & $-59: 36: 30.60$ & 320.21293 & -1.64975 & $Z Y J H K_{\mathrm{s}}$ & 4 \\
\hline d019 & $15: 24: 48.600$ & $-58: 49: 27.84$ & 321.67194 & -1.64978 & $Z Y J H K_{\mathrm{s}}$ & 4 \\
\hline d020 & $15: 33: 59.400$ & $-57: 59: 54.60$ & 323.13095 & -1.64976 & $Z Y J H K_{\mathrm{s}}$ & 4 \\
\hline $\mathrm{d} 021$ & $15: 42: 45.072$ & $-57: 08: 02.40$ & 324.58996 & -1.64971 & $Z Y J H K_{\mathrm{s}}$ & 4 \\
\hline $\mathrm{d} 022$ & 15:51:06.576 & $-56: 14: 02.40$ & 326.04898 & -1.64975 & $Z Y J H K_{\mathrm{s}}$ & 4 \\
\hline $\mathrm{d} 023$ & 15:59:04.920 & $-55: 18: 04.32$ & 327.50799 & -1.64974 & $Z Y J H K_{\mathrm{s}}$ & 5 \\
\hline $\mathrm{d} 024$ & $16: 06: 41.208$ & $-54: 20: 17.88$ & 328.96694 & -1.64974 & $Z Y J H K_{\mathrm{s}}$ & 5 \\
\hline $\mathrm{d} 025$ & $16: 13: 56.640$ & $-53: 20: 51.36$ & 330.42599 & -1.64974 & $Z Y J H K_{\mathrm{s}}$ & 5 \\
\hline $\mathrm{d} 026$ & $16: 20: 52.320$ & $-52: 19: 53.40$ & 331.88495 & -1.64978 & $Z Y J H K_{\mathrm{s}}$ & 5 \\
\hline $\mathrm{d} 027$ & $16: 27: 29.400$ & $-51: 17: 30.84$ & 333.34393 & -1.64976 & $J H K_{\mathrm{s}}$ & 2 \\
\hline $\mathrm{d} 028$ & $16: 33: 49.032$ & $-50: 13: 50.52$ & 334.80299 & -1.64976 & $J H K_{\mathrm{s}}$ & 2 \\
\hline d029 & $16: 39: 52.248$ & $-49: 08: 58.92$ & 336.26199 & -1.64971 & $J H K_{\mathrm{s}}$ & 2 \\
\hline $\mathrm{d} 030$ & $16: 45: 40.128$ & $-48: 03: 01.80$ & 337.72099 & -1.64973 & $Z Y J H K_{\mathrm{s}}$ & 2 \\
\hline $\mathrm{d} 031$ & $16: 51: 13.632$ & $-46: 56: 04.20$ & 339.17999 & -1.64971 & $Z Y J H K_{\mathrm{s}}$ & 2 \\
\hline d032 & $16: 56: 33.720$ & $-45: 48: 11.16$ & 340.63896 & -1.64975 & $Z Y J H K_{\mathrm{s}}$ & 2 \\
\hline $\mathrm{d} 033$ & $17: 01: 41.256$ & $-44: 39: 26.64$ & 342.09795 & -1.64972 & $Z Y J H K_{\mathrm{s}}$ & 1 \\
\hline $\mathrm{d} 034$ & $17: 06: 37.104$ & $-43: 29: 54.96$ & 343.55695 & -1.64975 & $Z Y J H K_{\mathrm{s}}$ & 1 \\
\hline d035 & $17: 11: 22.032$ & $-42: 19: 39.72$ & 345.01595 & -1.64979 & $Z Y J H K_{\mathrm{s}}$ & 1 \\
\hline $\mathrm{d} 036$ & $17: 15: 56.760$ & $-41: 08: 44.16$ & 346.47495 & -1.64979 & $J H K_{\mathrm{s}}$ & 1 \\
\hline $\mathrm{d} 037$ & $17: 20: 21.984$ & $-39: 57: 11.16$ & 347.93400 & -1.64973 & $J H K_{\mathrm{s}}$ & 1 \\
\hline d038 & $17: 24: 38.352$ & $-38: 45: 04.32$ & 349.39294 & -1.64975 & $J H K_{\mathrm{s}}$ & 1 \\
\hline $\mathrm{d} 039$ & $11: 45: 52.488$ & $-62: 28: 17.40$ & 295.43747 & -0.55759 & $Z Y J H K_{\mathrm{s}}$ & 5 \\
\hline $\mathrm{d} 040$ & $11: 58: 14.160$ & $-62: 48: 15.12$ & 296.89617 & -0.55758 & $Z Y J H K_{\mathrm{s}}$ & 5 \\
\hline d041 & $12: 10: 50.928$ & $-63: 04: 04.80$ & 298.35479 & -0.55753 & $Z Y J H K_{\mathrm{s}}$ & 5 \\
\hline $\mathrm{d} 042$ & $12: 23: 39.672$ & $-63: 15: 39.60$ & 299.81350 & -0.55756 & $Z Y J H K_{\mathrm{s}}$ & 5 \\
\hline $\mathrm{d} 043$ & $12: 36: 36.744$ & $-63: 22: 53.40$ & 301.27213 & -0.55754 & $Z Y J H K_{\mathrm{s}}$ & 5 \\
\hline d044 & $12: 49: 38.376$ & $-63: 25: 42.96$ & 302.73081 & -0.55755 & $Z Y J H K_{\mathrm{s}}$ & 5 \\
\hline $\mathrm{d} 045$ & $13: 02: 40.560$ & $-63: 24: 06.84$ & 304.18948 & -0.55760 & $Z Y J H K_{\mathrm{s}}$ & 5 \\
\hline $\mathrm{d} 046$ & $13: 15: 39.288$ & $-63: 18: 05.40$ & 305.64814 & -0.55754 & $Z Y J H K_{\mathrm{s}}$ & 5 \\
\hline $\mathrm{d} 047$ & $13: 28: 30.696$ & $-63: 07: 42.24$ & 307.10682 & -0.55756 & $J H K_{\mathrm{s}}$ & 5 \\
\hline $\mathrm{d} 048$ & $13: 41: 11.112$ & $-62: 53: 02.04$ & 308.56549 & -0.55756 & $J H K_{\mathrm{s}}$ & 5 \\
\hline d049 & $13: 53: 37.224$ & $-62: 34: 12.00$ & 310.02413 & -0.55760 & $Z Y J H K_{\mathrm{s}}$ & 5 \\
\hline $\mathrm{d} 050$ & $14: 05: 46.176$ & $-62: 11: 20.04$ & 311.48283 & -0.55756 & $Z Y J H K_{\mathrm{s}}$ & 5 \\
\hline d051 & $14: 17: 35.520$ & $-61: 44: 36.60$ & 312.94152 & -0.55757 & $Z Y J H K_{\mathrm{s}}$ & 5 \\
\hline $\mathrm{d} 052$ & 14:29:03.312 & $-61: 14: 12.48$ & 314.40014 & -0.55761 & $Z Y J H K_{\mathrm{s}}$ & 5 \\
\hline $\mathrm{d} 053$ & 14:40:08.135 & $-60: 40: 18.48$ & 315.85883 & -0.55752 & $Z Y J H K_{\mathrm{s}}$ & 4 \\
\hline d054 & $14: 50: 49.032$ & $-60: 03: 07.56$ & 317.31750 & -0.55758 & $Z Y J H K_{\mathrm{s}}$ & 4 \\
\hline $\mathrm{d} 055$ & $15: 01: 05.424$ & $-59: 22: 51.24$ & 318.77616 & -0.55761 & $Z Y J H K_{\mathrm{s}}$ & 4 \\
\hline d056 & $15: 10: 57.120$ & $-58: 39: 41.40$ & 320.23482 & -0.55759 & $Z Y J H K_{\mathrm{s}}$ & 3 \\
\hline $\mathrm{d} 057$ & $15: 20: 24.264$ & $-57: 53: 49.92$ & 321.69349 & -0.55755 & $Z Y J H K_{\mathrm{s}}$ & 4 \\
\hline
\end{tabular}


Table A.1. continued.

\begin{tabular}{|c|c|c|c|c|c|c|}
\hline $\begin{array}{l}\text { Tile } \\
\text { name }\end{array}$ & $\begin{array}{l}\text { RA (J2000.0) } \\
\text { dd:mm:ss.sss }\end{array}$ & $\begin{array}{c}\text { Dec (J2000.0) } \\
\text { dd:mm:ss.ss }\end{array}$ & $\begin{array}{c}\begin{array}{c}\text { Longitude } \\
\text { degrees }\end{array} \\
\text { deres }\end{array}$ & $\begin{array}{l}\text { Latitude } \\
\text { degrees }\end{array}$ & $\begin{array}{c}\text { Filters } \\
\text { completed }\end{array}$ & $\begin{array}{l}K_{\mathrm{s}} \text { epochs } \\
\text { completed }\end{array}$ \\
\hline $\mathrm{d} 058$ & $15: 29: 27.264$ & $-57: 05: 28.32$ & 323.15217 & -0.55754 & $Z Y J H K_{\mathrm{s}}$ & 4 \\
\hline $\mathrm{d} 059$ & $15: 38: 06.720$ & $-56: 14: 47.40$ & 324.61087 & -0.55755 & $Z Y J H K_{\mathrm{s}}$ & 4 \\
\hline $\mathrm{d} 060$ & $15: 46: 23.352$ & $-55: 21: 57.60$ & 326.06950 & -0.55754 & $Z Y J H K_{\mathrm{s}}$ & 4 \\
\hline $\mathrm{d} 061$ & $15: 54: 18.096$ & $-54: 27: 08.64$ & 327.52817 & -0.55761 & $Z Y J H K_{\mathrm{s}}$ & 5 \\
\hline $\mathrm{d} 062$ & $16: 01: 51.840$ & $-53: 30: 29.16$ & 328.98684 & -0.55756 & $Z Y J H K_{\mathrm{s}}$ & 5 \\
\hline $\mathrm{d} 063$ & 16:09:05.616 & $-52: 32: 08.16$ & 330.44549 & -0.55755 & $Z Y J H K_{\mathrm{s}}$ & 5 \\
\hline d064 & $16: 16: 00.456$ & $-51: 32: 13.20$ & 331.90419 & -0.55754 & $Z Y J H K_{\mathrm{s}}$ & 5 \\
\hline $\mathrm{d} 065$ & $16: 22: 37.368$ & $-50: 30: 51.84$ & 333.36286 & -0.55756 & $J H K_{\mathrm{s}}$ & 2 \\
\hline d066 & $16: 28: 57.360$ & $-49: 28: 10.56$ & 334.82153 & -0.55755 & $J H K_{\mathrm{s}}$ & 2 \\
\hline d067 & $16: 35: 01.416$ & $-48: 24: 15.84$ & 336.28015 & -0.55756 & $J H K_{\mathrm{s}}$ & 2 \\
\hline d068 & $16: 40: 50.520$ & $-47: 19: 13.08$ & 337.73882 & -0.55761 & $Z Y J H K_{\mathrm{s}}$ & 2 \\
\hline $\mathrm{d} 069$ & $16: 46: 25.560$ & $-46: 13: 07.32$ & 339.19753 & -0.55758 & $Z Y J H K_{\mathrm{s}}$ & 2 \\
\hline $\mathrm{d} 070$ & $16: 51: 47.400$ & $-45: 06: 03.96$ & 340.65613 & -0.55757 & $Z Y J H K_{\mathrm{s}}$ & 2 \\
\hline $\mathrm{d} 071$ & $16: 56: 56.928$ & $-43: 58: 06.96$ & 342.11483 & -0.55761 & $Z Y J H K_{\mathrm{s}}$ & 1 \\
\hline $\mathrm{d} 072$ & $17: 01: 54.864$ & $-42: 49: 20.28$ & 343.57351 & -0.55754 & $Z Y J H K_{\mathrm{s}}$ & 1 \\
\hline d073 & 17:06:42.000 & $-41: 39: 48.24$ & 345.03215 & -0.55756 & $Z Y J H K_{\mathrm{s}}$ & 1 \\
\hline d074 & $17: 11: 19.032$ & $-40: 29: 33.72$ & 346.49083 & -0.55759 & $J H K_{\mathrm{s}}$ & 1 \\
\hline $\mathrm{d} 075$ & $17: 15: 46.608$ & $-39: 18: 39.96$ & 347.94953 & -0.55759 & $J H K_{\mathrm{s}}$ & 1 \\
\hline d076 & $17: 20: 05.328$ & $-38: 07: 09.84$ & 349.40822 & -0.55752 & $J H K_{\mathrm{s}}$ & 1 \\
\hline d077 & 11:48:10.080 & $-61: 24: 47.16$ & 295.43749 & 0.53461 & $Z Y J H K_{\mathrm{s}}$ & 5 \\
\hline $\mathrm{d} 078$ & 12:00:07.584 & $-61: 44: 03.84$ & 296.89636 & 0.53458 & $Z Y J H K_{\mathrm{s}}$ & 5 \\
\hline d079 & $12: 12: 18.648$ & $-61: 59: 20.40$ & 298.35521 & 0.53456 & $Z Y J H K_{\mathrm{s}}$ & 5 \\
\hline d080 & $12: 24: 40.392$ & $-62: 10: 30.00$ & 299.81408 & 0.53461 & $Z Y J H K_{\mathrm{s}}$ & 5 \\
\hline $\mathrm{d} 081$ & $12: 37: 09.600$ & $-62: 17: 27.96$ & 301.27295 & 0.53465 & $Z Y J H K_{\mathrm{s}}$ & 5 \\
\hline $\mathrm{d} 082$ & $12: 49: 42.840$ & $-62: 20: 11.40$ & 302.73182 & 0.53458 & $Z Y J H K_{\mathrm{s}}$ & 5 \\
\hline d083 & $13: 02: 16.560$ & $-62: 18: 38.16$ & 304.19066 & 0.53466 & $Z Y J H K_{\mathrm{s}}$ & 5 \\
\hline $\mathrm{d} 084$ & $13: 14: 47.232$ & $-62: 12: 50.04$ & 305.64955 & 0.53458 & $Z Y J H K_{\mathrm{s}}$ & 5 \\
\hline $\mathrm{d} 085$ & $13: 27: 11.328$ & $-62: 02: 48.84$ & 307.10837 & 0.53460 & $Z Y J H K_{\mathrm{s}}$ & 4 \\
\hline d086 & $13: 39: 25.632$ & $-61: 48: 39.24$ & 308.56725 & 0.53465 & $Z Y J H K_{\mathrm{s}}$ & 4 \\
\hline $\mathrm{d} 087$ & $13: 51: 27.144$ & $-61: 30: 28.08$ & 310.02613 & 0.53456 & $Z Y J H K_{\mathrm{s}}$ & 5 \\
\hline $\mathrm{d} 088$ & $14: 03: 13.176$ & $-61: 08: 22.20$ & 311.48497 & 0.53458 & $Z Y J H K_{\mathrm{s}}$ & 5 \\
\hline d089 & $14: 14: 41.544$ & $-60: 42: 30.60$ & 312.94386 & 0.53465 & $Z Y J H K_{\mathrm{s}}$ & 4 \\
\hline d090 & $14: 25: 50.400$ & $-60: 13: 03.72$ & 314.40272 & 0.53463 & $Z Y J H K_{\mathrm{s}}$ & 4 \\
\hline d091 & $14: 36: 38.352$ & $-59: 40: 11.64$ & 315.86159 & 0.53462 & $Z Y J H K_{\mathrm{s}}$ & 4 \\
\hline d092 & 14:47:04.392 & $-59: 04: 05.52$ & 317.32043 & 0.53461 & $Z Y J H K_{\mathrm{s}}$ & 4 \\
\hline d093 & 14:57:07.920 & $-58: 24: 56.16$ & 318.77932 & 0.53465 & $Z Y J H K_{\mathrm{s}}$ & 3 \\
\hline d094 & $15: 06: 48.648$ & $-57: 42: 55.44$ & 320.23818 & 0.53459 & $Z Y J H K_{\mathrm{s}}$ & 3 \\
\hline d095 & 15:16:06.552 & $-56: 58: 13.80$ & 321.69700 & 0.53459 & $Z Y J H K_{\mathrm{s}}$ & 5 \\
\hline d096 & $15: 25: 01.920$ & $-56: 11: 02.04$ & 323.15588 & 0.53461 & $Z Y J H K_{\mathrm{s}}$ & 5 \\
\hline d097 & $15: 33: 35.208$ & $-55: 21: 30.60$ & 324.61479 & 0.53459 & $Z Y J H K_{\mathrm{s}}$ & 4 \\
\hline d098 & $15: 41: 46.968$ & $-54: 29: 49.20$ & 326.07365 & 0.53465 & $Z Y J H K_{\mathrm{s}}$ & 4 \\
\hline d099 & $15: 49: 37.968$ & $-53: 36: 07.56$ & 327.53250 & 0.53463 & $Z Y J H K_{\mathrm{s}}$ & 4 \\
\hline $\mathrm{d} 100$ & $15: 57: 09.024$ & $-52: 40: 34.32$ & 328.99135 & 0.53458 & $Z Y J H K_{\mathrm{s}}$ & 4 \\
\hline d101 & 16:04:20.976 & $-51: 43: 17.40$ & 330.45023 & 0.53459 & $Z Y J H K_{\mathrm{s}}$ & 5 \\
\hline d102 & $16: 11: 14.736$ & $-50: 44: 24.72$ & 331.90911 & 0.53460 & $Z Y J H K_{\mathrm{s}}$ & 5 \\
\hline d103 & $16: 17: 51.216$ & $-49: 44: 03.48$ & 333.36798 & 0.53460 & $Z Y J H K_{\mathrm{s}}$ & 3 \\
\hline d104 & $16: 24: 11.328$ & $-48: 42: 20.16$ & 334.82687 & 0.53461 & $Z Y J H K_{\mathrm{s}}$ & 3 \\
\hline d105 & $16: 30: 15.960$ & $-47: 39: 21.24$ & 336.28568 & 0.53460 & $Z Y J H K_{\mathrm{s}}$ & 3 \\
\hline d106 & $16: 36: 06.000$ & $-46: 35: 12.12$ & 337.74450 & 0.53460 & $Z Y J H K_{\mathrm{s}}$ & 3 \\
\hline d107 & $16: 41: 42.312$ & $-45: 29: 57.84$ & 339.20338 & 0.53460 & $J H K_{\mathrm{s}}$ & 1 \\
\hline d108 & $16: 47: 05.712$ & $-44: 23: 43.44$ & 340.66229 & 0.53457 & $J H K_{\mathrm{s}}$ & 1 \\
\hline d109 & $16: 52: 16.944$ & $-43: 16: 33.24$ & 342.12118 & 0.53461 & $J H K_{\mathrm{s}}$ & 1 \\
\hline d110 & $16: 57: 16.776$ & $-42: 08: 31.56$ & 343.58005 & 0.53462 & $Z Y J H K_{\mathrm{s}}$ & 1 \\
\hline d111 & 17:02:05.904 & $-40: 59: 42.36$ & 345.03883 & 0.53459 & $Z Y J H K_{\mathrm{s}}$ & 1 \\
\hline $\mathrm{d} 112$ & $17: 06: 45.024$ & $-39: 50: 08.52$ & 346.49771 & 0.53457 & $Z Y J H K_{\mathrm{s}}$ & 1 \\
\hline d113 & 17:11:14.736 & $-38: 39: 53.28$ & 347.95664 & 0.53461 & $Z Y J H K_{\mathrm{s}}$ & 2 \\
\hline d114 & $17: 15: 35.640$ & $-37: 29: 00.24$ & 349.41546 & 0.53460 & $Z Y J H K_{\mathrm{s}}$ & 2 \\
\hline d115 & 11:50:18.720 & $-60: 21: 09.00$ & 295.43768 & 1.62680 & $Z Y J H K_{\mathrm{s}}$ & 5 \\
\hline
\end{tabular}


Table A.1. continued.

\begin{tabular}{|c|c|c|c|c|c|c|}
\hline $\begin{array}{l}\text { Tile } \\
\text { name }\end{array}$ & $\begin{array}{l}\text { RA (J2000.0) } \\
\text { dd:mm:ss.sss }\end{array}$ & $\begin{array}{c}\text { Dec (J2000.0) } \\
\text { dd:mm:ss.ss }\end{array}$ & $\begin{array}{c}\text { Longitude } \\
\text { degrees }\end{array}$ & $\begin{array}{l}\text { Latitude } \\
\text { degrees }\end{array}$ & $\begin{array}{c}\text { Filters } \\
\text { completed }\end{array}$ & $\begin{array}{l}K_{\mathrm{S}} \text { epochs } \\
\text { completed }\end{array}$ \\
\hline $\mathrm{d} 116$ & $12: 01: 53.760$ & $-60: 39: 47.52$ & 296.89732 & 1.62677 & $Z Y J H K_{\mathrm{s}}$ & 5 \\
\hline d117 & 12:13:40.992 & $-60: 54: 32.75$ & 298.35689 & 1.62684 & $Z Y J H K_{\mathrm{s}}$ & 5 \\
\hline d11 18 & $12: 25: 37.800$ & $-61: 05: 19.68$ & 299.81648 & 1.62674 & $Z Y J H K_{\mathrm{s}}$ & 5 \\
\hline d11 19 & $12: 37: 41.304$ & $-61: 12: 02.52$ & 301.27608 & 1.62684 & $Z Y J H K_{\mathrm{s}}$ & 5 \\
\hline d120 & $12: 49: 48.408$ & $-61: 14: 39.48$ & 302.73567 & 1.62683 & $Z Y J H K_{\mathrm{s}}$ & 5 \\
\hline $\mathrm{d} 121$ & 13:01:55.944 & $-61: 13: 09.12$ & 304.19526 & 1.62680 & $Z Y J H K_{\mathrm{s}}$ & 5 \\
\hline $\mathrm{d} 122$ & $13: 14: 00.720$ & $-61: 07: 32.15$ & 305.65484 & 1.62675 & $Z Y J H K_{\mathrm{s}}$ & 5 \\
\hline d123 & $13: 25: 59.640$ & $-60: 57: 50.40$ & 307.11447 & 1.62681 & $Z Y J H K_{\mathrm{s}}$ & 4 \\
\hline d124 & $13: 37: 49.704$ & $-60: 44: 08.88$ & 308.57402 & 1.62675 & $Z Y J H K_{\mathrm{s}}$ & 4 \\
\hline $\mathrm{d} 125$ & $13: 49: 28.248$ & $-60: 26: 32.28$ & 310.03361 & 1.62680 & $Z Y J H K_{\mathrm{s}}$ & 5 \\
\hline d126 & 14:00:52.872 & $-60: 05: 08.16$ & 311.49324 & 1.62678 & $Z Y J H K_{\mathrm{s}}$ & 5 \\
\hline d127 & $14: 12: 01.440$ & $-59: 40: 04.44$ & 312.95280 & 1.62677 & $Z Y J H K_{\mathrm{s}}$ & 4 \\
\hline $\mathrm{d} 128$ & $14: 22: 52.272$ & $-59: 11: 29.76$ & 314.41240 & 1.62684 & $Z Y J H K_{\mathrm{s}}$ & 4 \\
\hline d129 & $14: 33: 24.024$ & $-58: 39: 34.56$ & 315.87197 & 1.62678 & $Z Y J H K_{\mathrm{s}}$ & 4 \\
\hline d130 & $14: 43: 35.688$ & $-58: 04: 28.20$ & 317.33156 & 1.62679 & $Z Y J H K_{\mathrm{s}}$ & 4 \\
\hline d131 & $14: 53: 26.616$ & $-57: 26: 21.48$ & 318.79117 & 1.62676 & $Z Y J H K_{\mathrm{s}}$ & 3 \\
\hline d132 & $15: 02: 56.424$ & $-56: 45: 24.48$ & 320.25077 & 1.62679 & $Z Y J H K_{\mathrm{s}}$ & 3 \\
\hline d133 & $15: 12: 05.040$ & $-56: 01: 48.00$ & 321.71037 & 1.62674 & $Z Y J H K_{\mathrm{s}}$ & 5 \\
\hline d134 & $15: 20: 52.560$ & $-55: 15: 41.76$ & 323.16993 & 1.62676 & $Z Y J H K_{\mathrm{s}}$ & 5 \\
\hline d135 & $15: 29: 19.344$ & $-54: 27: 15.48$ & 324.62955 & 1.62682 & $Z Y J H K_{\mathrm{s}}$ & 4 \\
\hline d136 & $15: 37: 25.872$ & $-53: 36: 39.24$ & 326.08912 & 1.62676 & $Z Y J H K_{\mathrm{s}}$ & 4 \\
\hline d137 & $15: 45: 12.720$ & $-52: 44: 01.32$ & 327.54872 & 1.62676 & $Z Y J H K_{\mathrm{s}}$ & 4 \\
\hline d138 & $15: 52: 40.584$ & $-51: 49: 30.36$ & 329.00835 & 1.62678 & $Z Y J H K_{\mathrm{s}}$ & 4 \\
\hline d139 & $15: 59: 50.184$ & $-50: 53: 14.64$ & 330.46790 & 1.62680 & $Z Y J H K_{\mathrm{s}}$ & 5 \\
\hline d140 & $16: 06: 42.360$ & $-49: 55: 21.36$ & 331.92752 & 1.62681 & $Z Y J H K_{\mathrm{s}}$ & 5 \\
\hline d141 & $16: 13: 17.904$ & $-48: 55: 57.72$ & 333.38714 & 1.62678 & $Z Y J H K_{\mathrm{s}}$ & 3 \\
\hline d142 & $16: 19: 37.608$ & $-47: 55: 10.20$ & 334.84670 & 1.62678 & $Z Y J H K_{\mathrm{s}}$ & 3 \\
\hline d143 & $16: 25: 42.312$ & $-46: 53: 04.92$ & 336.30624 & 1.62678 & $Z Y J H K_{\mathrm{s}}$ & 3 \\
\hline d144 & $16: 31: 32.832$ & $-45: 49: 46.92$ & 337.76590 & 1.62684 & $Z Y J H K_{\mathrm{s}}$ & 3 \\
\hline d145 & $16: 37: 09.936$ & $-44: 45: 22.32$ & 339.22547 & 1.62682 & $Z Y J H K_{\mathrm{s}}$ & 1 \\
\hline d146 & $16: 42: 34.392$ & $-43: 39: 55.44$ & 340.68506 & 1.62681 & $J H K_{\mathrm{s}}$ & 1 \\
\hline d147 & $16: 47: 46.920$ & $-42: 33: 30.96$ & 342.14462 & 1.62677 & $J H K_{\mathrm{s}}$ & 1 \\
\hline d148 & $16: 52: 48.216$ & $-41: 26: 12.48$ & 343.60424 & 1.62683 & $J H K_{\mathrm{s}}$ & 1 \\
\hline d149 & $16: 57: 38.976$ & $-40: 18: 04.32$ & 345.06387 & 1.62680 & $Z Y J H K_{\mathrm{s}}$ & 1 \\
\hline d150 & $17: 02: 19.800$ & $-39: 09: 10.08$ & 346.52343 & 1.62677 & $Z Y J H K_{\mathrm{s}}$ & 1 \\
\hline d151 & 17:06:51.312 & $-37: 59: 32.64$ & 347.98303 & 1.62675 & $Z Y J H K_{\mathrm{s}}$ & 2 \\
\hline d152 & 17:11:14.064 & $-36: 49: 15.24$ & 349.44260 & 1.62674 & $Z Y J H K_{\mathrm{s}}$ & 2 \\
\hline b201 & 18:04:24.384 & $-41: 44: 53.52$ & 350.74816 & -9.68974 & $Z Y J H K_{\mathrm{s}}$ & 1 \\
\hline b202 & 18:08:00.144 & $-40: 27: 29.88$ & 352.22619 & -9.68971 & $Z Y J H K_{\mathrm{s}}$ & 1 \\
\hline b203 & 18:11:29.496 & $-39: 09: 52.92$ & 353.70409 & -9.68973 & $Z Y J H K_{\mathrm{s}}$ & 1 \\
\hline b204 & 18:14:52.992 & $-37: 52: 03.36$ & 355.18207 & -9.68974 & $Z Y J H K_{\mathrm{s}}$ & 1 \\
\hline b205 & 18:18:11.136 & $-36: 34: 02.64$ & 356.66012 & -9.68976 & $Z Y J H K_{\mathrm{s}}$ & 1 \\
\hline b206 & $18: 21: 24.360$ & $-35: 15: 52.20$ & 358.13813 & -9.68975 & $Z Y J H K_{\mathrm{s}}$ & 1 \\
\hline b207 & $18: 24: 33.096$ & $-33: 57: 33.48$ & 359.61607 & -9.68977 & $Z Y J H K_{\mathrm{s}}$ & 2 \\
\hline b208 & $18: 27: 37.728$ & $-32: 39: 07.20$ & 1.09399 & -9.68974 & $Z Y J H K_{\mathrm{s}}$ & 2 \\
\hline b209 & 18:30:38.640 & $-31: 20: 34.08$ & 2.57200 & -9.68971 & $Z Y J H K_{\mathrm{s}}$ & 2 \\
\hline b210 & $18: 33: 36.168$ & $-30: 01: 55.56$ & 4.04998 & -9.68973 & $Z Y J H K_{\mathrm{s}}$ & 2 \\
\hline b211 & $18: 36: 30.624$ & $-28: 43: 12.36$ & 5.52796 & -9.68978 & $Z Y J H K_{\mathrm{s}}$ & 2 \\
\hline b212 & $18: 39: 22.272$ & $-27: 24: 25.20$ & 7.00593 & -9.68975 & $Z Y J H K_{\mathrm{s}}$ & 3 \\
\hline b213 & $18: 42: 11.424$ & $-26: 05: 34.80$ & 8.48396 & -9.68974 & $Z Y J H K_{\mathrm{s}}$ & 2 \\
\hline b214 & $18: 44: 58.320$ & $-24: 46: 42.24$ & 9.96193 & -9.68974 & $Z Y J H K_{\mathrm{s}}$ & 3 \\
\hline b215 & $17: 59: 15.960$ & $-41: 13: 55.92$ & 350.74595 & -8.59756 & $Z Y J H K_{\mathrm{s}}$ & 1 \\
\hline b216 & 18:02:55.992 & $-39: 57: 07.92$ & 352.21956 & -8.59753 & $Z Y J H K_{\mathrm{s}}$ & 1 \\
\hline b217 & 18:06:29.472 & $-38: 40: 04.08$ & 353.69327 & -8.59756 & $Z Y J H K_{\mathrm{s}}$ & 1 \\
\hline b218 & 18:09:56.880 & $-37: 22: 46.56$ & 355.16684 & -8.59757 & $Z Y J H K_{\mathrm{s}}$ & 1 \\
\hline b219 & 18:13:18.768 & $-36: 05: 16.07$ & 356.64051 & -8.59760 & $Z Y J H K_{\mathrm{s}}$ & 1 \\
\hline b220 & $18: 16: 35.568$ & $-34: 47: 34.08$ & 358.11423 & -8.59759 & $Z Y J H K_{\mathrm{s}}$ & 1 \\
\hline b221 & 18:19:47.688 & $-33: 29: 42.36$ & 359.58781 & -8.59757 & $Z Y J H K_{\mathrm{s}}$ & 2 \\
\hline
\end{tabular}


Table A.1. continued.

\begin{tabular}{|c|c|c|c|c|c|c|}
\hline $\begin{array}{l}\text { Tile } \\
\text { name }\end{array}$ & $\begin{array}{l}\text { RA (J2000.0) } \\
\text { dd:mm:ss.sss }\end{array}$ & $\begin{array}{c}\text { Dec (J2000.0) } \\
\text { dd:mm:ss.ss }\end{array}$ & $\begin{array}{c}\begin{array}{c}\text { Longitude } \\
\text { degrees }\end{array} \\
\text { deres }\end{array}$ & $\begin{array}{l}\text { Latitude } \\
\text { degrees }\end{array}$ & $\begin{array}{c}\text { Filters } \\
\text { completed }\end{array}$ & $\begin{array}{l}K_{\mathrm{s}} \text { epochs } \\
\text { completed }\end{array}$ \\
\hline $\mathrm{b} 222$ & $18: 22: 55.560$ & $-32: 11: 41.28$ & 1.06151 & -8.59755 & $Z Y J H K_{\mathrm{s}}$ & 2 \\
\hline b223 & $18: 25: 59.544$ & $-30: 53: 32.28$ & 2.53522 & -8.59757 & $Z Y J H K_{\mathrm{s}}$ & 2 \\
\hline b224 & $18: 28: 59.952$ & $-29: 35: 16.80$ & 4.00880 & -8.59759 & $Z Y J H K_{\mathrm{s}}$ & 2 \\
\hline b225 & $18: 31: 57.120$ & $-28: 16: 54.84$ & 5.48250 & -8.59755 & $Z Y J H K_{\mathrm{s}}$ & 2 \\
\hline b226 & $18: 34: 51.360$ & $-26: 58: 27.84$ & 6.95620 & -8.59757 & $Z Y J H K_{\mathrm{s}}$ & 3 \\
\hline b227 & $18: 37: 42.912$ & $-25: 39: 56.88$ & 8.42977 & -8.59756 & $Z Y J H K_{\mathrm{s}}$ & 2 \\
\hline b228 & $18: 40: 32.088$ & $-24: 21: 21.96$ & 9.90350 & -8.59757 & $Z Y J H K_{\mathrm{s}}$ & 3 \\
\hline b229 & $17: 54: 12.456$ & $-40: 42: 07.56$ & 350.74383 & -7.50542 & $Y J H K_{\mathrm{s}}$ & 1 \\
\hline b230 & $17: 57: 56.496$ & $-39: 25: 54.48$ & 352.21380 & -7.50537 & $J H K_{\mathrm{s}}$ & - \\
\hline b231 & 18:01:33.792 & $-38: 09: 24.48$ & 353.68363 & -7.50537 & $J H K_{\mathrm{s}}$ & 1 \\
\hline b232 & 18:05:04.920 & $-36: 52: 38.28$ & 355.15359 & -7.50541 & $J H K_{\mathrm{s}}$ & 1 \\
\hline b233 & 18:08:30.312 & $-35: 35: 38.04$ & 356.62342 & -7.50539 & $J H K_{\mathrm{s}}$ & 1 \\
\hline b234 & $18: 11: 50.472$ & $-34: 18: 24.48$ & 358.09337 & -7.50535 & $J H K_{\mathrm{s}}$ & 1 \\
\hline b235 & $18: 15: 05.832$ & $-33: 00: 59.76$ & 359.56322 & -7.50542 & $J H K_{\mathrm{s}}$ & 2 \\
\hline b236 & $18: 18: 16.752$ & $-31: 43: 24.24$ & 1.03312 & -7.50538 & $Z Y J H K_{\mathrm{s}}$ & 2 \\
\hline b237 & $18: 21: 23.640$ & $-30: 25: 39.36$ & 2.50307 & -7.50541 & $Z Y J H K_{\mathrm{s}}$ & 2 \\
\hline b238 & $18: 24: 26.808$ & $-29: 07: 46.20$ & 3.97300 & -7.50540 & $J H K_{\mathrm{s}}$ & 2 \\
\hline b239 & $18: 27: 26.568$ & $-27: 49: 45.84$ & 5.44287 & -7.50536 & $J H K_{\mathrm{s}}$ & 2 \\
\hline b240 & $18: 30: 23.256$ & $-26: 31: 39.36$ & 6.91271 & -7.50540 & $J H K_{\mathrm{s}}$ & 2 \\
\hline b241 & 18:33:17.136 & $-25: 13: 27.12$ & 8.38261 & -7.50541 & $J H K_{\mathrm{s}}$ & 3 \\
\hline b242 & $18: 36: 08.472$ & $-23: 55: 10.20$ & 9.85251 & -7.50542 & $J H K_{\mathrm{s}}$ & 2 \\
\hline b243 & $17: 49: 13.848$ & $-40: 09: 29.16$ & 350.74206 & -6.41324 & $Y J H K_{\mathrm{s}}$ & 1 \\
\hline b244 & 17:53:01.608 & $-38: 53: 51.72$ & 352.20875 & -6.41323 & $J H K_{\mathrm{s}}$ & - \\
\hline b245 & $17: 56: 42.504$ & $-37: 37: 54.84$ & 353.67546 & -6.41323 & $J H K_{\mathrm{s}}$ & 1 \\
\hline b246 & 18:00:17.064 & $-36: 21: 40.32$ & 355.14219 & -6.41321 & $J H K_{\mathrm{s}}$ & 1 \\
\hline b247 & 18:03:45.792 & $-35: 05: 09.96$ & 356.60888 & -6.41323 & $J H K_{\mathrm{s}}$ & 1 \\
\hline b248 & 18:07:09.120 & $-33: 48: 25.20$ & 358.07550 & -6.41322 & $J H K_{\mathrm{s}}$ & 1 \\
\hline b249 & 18:10:27.504 & $-32: 31: 27.12$ & 359.54218 & -6.41323 & $J H K_{\mathrm{s}}$ & 2 \\
\hline b250 & $18: 13: 41.328$ & $-31: 14: 17.16$ & 1.00886 & -6.41325 & $Z Y J H K_{\mathrm{s}}$ & 2 \\
\hline b251 & $18: 16: 50.952$ & $-29: 56: 56.04$ & 2.47562 & -6.41319 & $J H K_{\mathrm{s}}$ & 2 \\
\hline b252 & 18:19:56.736 & $-28: 39: 25.92$ & 3.94224 & -6.41326 & $J H K_{\mathrm{s}}$ & 2 \\
\hline b253 & $18: 22: 58.968$ & $-27: 21: 46.80$ & 5.40892 & -6.41319 & $J H K_{\mathrm{s}}$ & 2 \\
\hline b254 & $18: 25: 58.008$ & $-26: 04: 00.12$ & 6.87563 & -6.41325 & $J H K_{\mathrm{s}}$ & 2 \\
\hline b255 & $18: 28: 54.072$ & $-24: 46: 06.60$ & 8.34231 & -6.41319 & $J H K_{\mathrm{s}}$ & 3 \\
\hline b256 & $18: 31: 47.496$ & $-23: 28: 07.32$ & 9.80903 & -6.41325 & $J H K_{\mathrm{s}}$ & 2 \\
\hline b257 & $17: 44: 20.112$ & $-39: 36: 02.16$ & 350.74076 & -5.32104 & $J H K_{\mathrm{s}}$ & 1 \\
\hline b258 & $17: 48: 11.328$ & $-38: 20: 59.64$ & 352.20485 & -5.32102 & $J H K_{\mathrm{s}}$ & 1 \\
\hline b259 & $17: 51: 55.560$ & $-37: 05: 36.24$ & 353.66885 & -5.32101 & $J H K_{\mathrm{s}}$ & - \\
\hline b260 & $17: 55: 33.360$ & $-35: 49: 53.40$ & 355.13291 & -5.32104 & $J H K_{\mathrm{s}}$ & - \\
\hline b261 & 17:59:05.184 & $-34: 33: 52.92$ & 356.59692 & -5.32103 & $J H K_{\mathrm{s}}$ & - \\
\hline b262 & $18: 02: 31.512$ & $-33: 17: 36.24$ & 358.06096 & -5.32105 & $J H K_{\mathrm{s}}$ & - \\
\hline b263 & 18:05:52.752 & $-32: 01: 04.80$ & 359.52500 & -5.32104 & $J H K_{\mathrm{s}}$ & - \\
\hline b264 & 18:09:09.288 & $-30: 44: 20.04$ & 0.98899 & -5.32099 & $J H K_{\mathrm{s}}$ & - \\
\hline b265 & $18: 12: 21.528$ & $-29: 27: 23.40$ & 2.45295 & -5.32106 & $J H K_{\mathrm{s}}$ & - \\
\hline b266 & $18: 15: 29.784$ & $-28: 10: 15.24$ & 3.91703 & -5.32105 & $J H K_{\mathrm{s}}$ & - \\
\hline b267 & $18: 18: 34.368$ & $-26: 52: 57.36$ & 5.38103 & -5.32101 & $J H K_{\mathrm{s}}$ & - \\
\hline b268 & $18: 21: 35.616$ & $-25: 35: 30.48$ & 6.84507 & -5.32101 & $J H K_{\mathrm{s}}$ & - \\
\hline b269 & $18: 24: 33.792$ & $-24: 17: 55.68$ & 8.30909 & -5.32100 & $J H K_{\mathrm{s}}$ & 1 \\
\hline b270 & $18: 27: 29.184$ & $-23: 00: 14.04$ & 9.77309 & -5.32107 & $Z Y J H K_{\mathrm{s}}$ & 1 \\
\hline b271 & $17: 39: 31.128$ & $-39: 01: 49.44$ & 350.73953 & -4.22883 & $J H K_{\mathrm{s}}$ & 1 \\
\hline b272 & $17: 43: 25.536$ & $-37: 47: 22.20$ & 352.20141 & -4.22884 & $J H K_{\mathrm{s}}$ & 1 \\
\hline b273 & $17: 47: 12.888$ & $-36: 32: 31.92$ & 353.66332 & -4.22886 & $J H K_{\mathrm{s}}$ & 1 \\
\hline b274 & $17: 50: 53.688$ & $-35: 17: 20.76$ & 355.12516 & -4.22890 & $J H K_{\mathrm{s}}$ & 1 \\
\hline b275 & $17: 54: 28.416$ & $-34: 01: 49.80$ & 356.58709 & -4.22886 & $J H K_{\mathrm{s}}$ & 1 \\
\hline b276 & $17: 57: 57.528$ & $-32: 46: 01.20$ & 358.04898 & -4.22882 & $J H K_{\mathrm{s}}$ & 1 \\
\hline b277 & 18:01:21.456 & $-31: 29: 56.40$ & 359.51088 & -4.22881 & $J H K_{\mathrm{s}}$ & 1 \\
\hline b278 & 18:04:40.584 & $-30: 13: 36.84$ & 0.97275 & -4.22884 & $J H K_{\mathrm{s}}$ & 1 \\
\hline b279 & 18:07:55.272 & $-28: 57: 03.60$ & 2.43463 & -4.22884 & $J H K_{\mathrm{s}}$ & 1 \\
\hline
\end{tabular}


Table A.1. continued.

\begin{tabular}{|c|c|c|c|c|c|c|}
\hline $\begin{array}{l}\text { Tile } \\
\text { name }\end{array}$ & $\begin{array}{l}\text { RA (J2000.0) } \\
\text { dd:mm:ss.sss }\end{array}$ & $\begin{array}{c}\text { Dec (J2000.0) } \\
\text { dd:mm:ss.ss }\end{array}$ & $\begin{array}{c}\text { Longitude } \\
\text { degrees }\end{array}$ & $\begin{array}{l}\text { Latitude } \\
\text { degrees }\end{array}$ & $\begin{array}{c}\text { Filters } \\
\text { completed }\end{array}$ & $\begin{array}{l}K_{\mathrm{S}} \text { epochs } \\
\text { completed }\end{array}$ \\
\hline b280 & $18: 11: 05.880$ & $-27: 40: 17.76$ & 3.89659 & -4.22886 & $J H K_{\mathrm{s}}$ & 1 \\
\hline b281 & $18: 14: 12.696$ & $-26: 23: 20.76$ & 5.35849 & -4.22883 & $J H K_{\mathrm{s}}$ & 1 \\
\hline b282 & $18: 17: 16.056$ & $-25: 06: 13.68$ & 6.82039 & -4.22886 & $J H K_{\mathrm{s}}$ & 1 \\
\hline b283 & $18: 20: 16.224$ & $-23: 48: 57.60$ & 8.28222 & -4.22888 & $J H K_{\mathrm{s}}$ & - \\
\hline b284 & $18: 23: 13.488$ & $-22: 31: 32.88$ & 9.74416 & -4.22889 & $J H K_{\mathrm{s}}$ & - \\
\hline b285 & $17: 34: 46.896$ & $-38: 26: 51.72$ & 350.73871 & -3.13666 & $J H K_{\mathrm{s}}$ & - \\
\hline b286 & $17: 38: 44.256$ & $-37: 12: 59.40$ & 352.19896 & -3.13670 & $J H K_{\mathrm{s}}$ & - \\
\hline b287 & $17: 42: 34.488$ & $-35: 58: 41.88$ & 353.65931 & -3.13670 & $J H K_{\mathrm{s}}$ & - \\
\hline b288 & $17: 46: 18.096$ & $-34: 44: 01.68$ & 355.11962 & -3.13673 & $Z Y J H K_{\mathrm{s}}$ & - \\
\hline b289 & $17: 49: 55.536$ & $-33: 29: 00.24$ & 356.57994 & -3.13668 & $Z Y J H K_{\mathrm{s}}$ & 1 \\
\hline b290 & $17: 53: 27.288$ & $-32: 13: 39.72$ & 358.04023 & -3.13673 & $Z Y J H K_{\mathrm{s}}$ & 1 \\
\hline b291 & $17: 56: 53.736$ & $-30: 58: 01.20$ & 359.50054 & -3.13672 & $Z Y J H K_{\mathrm{s}}$ & - \\
\hline b292 & $18: 00: 15.264$ & $-29: 42: 06.12$ & 0.96088 & -3.13663 & $Z Y$ & - \\
\hline b293 & 18:03:32.280 & $-28: 25: 56.28$ & 2.42120 & -3.13666 & $Z Y$ & - \\
\hline b294 & $18: 06: 45.096$ & $-27: 09: 32.75$ & 3.88150 & -3.13671 & $Z Y J H K_{\mathrm{s}}$ & - \\
\hline b295 & $18: 09: 54.024$ & $-25: 52: 56.64$ & 5.34179 & -3.13672 & $Z Y J H K_{\mathrm{s}}$ & - \\
\hline b296 & $18: 12: 59.352$ & $-24: 36: 09.00$ & 6.80204 & -3.13666 & $Z Y J H K_{\mathrm{s}}$ & - \\
\hline b297 & 18:16:01.416 & $-23: 19: 10.92$ & 8.26235 & -3.13668 & $Z Y J H K_{\mathrm{s}}$ & 1 \\
\hline b298 & $18: 19: 00.456$ & $-22: 02: 03.12$ & 9.72271 & -3.13666 & $J H K_{\mathrm{s}}$ & 1 \\
\hline b299 & 17:30:07.272 & $-37: 51: 11.88$ & 350.73789 & -2.04453 & $J H K_{\mathrm{s}}$ & - \\
\hline b300 & $17: 34: 07.344$ & $-36: 37: 53.76$ & 352.19711 & -2.04451 & $J H K_{\mathrm{s}}$ & - \\
\hline b301 & $17: 38: 00.240$ & $-35: 24: 09.00$ & 353.65635 & -2.04453 & $Z Y J H K_{\mathrm{s}}$ & - \\
\hline b302 & $17: 41: 46.440$ & $-34: 09: 59.40$ & 355.11565 & -2.04449 & $Z Y J H K_{\mathrm{s}}$ & - \\
\hline b303 & $17: 45: 26.424$ & $-32: 55: 27.48$ & 356.57487 & -2.04453 & $Z Y J H K_{\mathrm{s}}$ & 1 \\
\hline b304 & $17: 49: 00.600$ & $-31: 40: 34.32$ & 358.03411 & -2.04446 & $Z Y J H K_{\mathrm{s}}$ & 1 \\
\hline b305 & $17: 52: 29.424$ & $-30: 25: 22.08$ & 359.49334 & -2.04452 & $Z Y J H K_{\mathrm{s}}$ & - \\
\hline b306 & $17: 55: 53.256$ & $-29: 09: 51.84$ & 0.95261 & -2.04456 & $Z Y J H K_{\mathrm{s}}$ & - \\
\hline b307 & $17: 59: 12.432$ & $-27: 54: 05.04$ & 2.41186 & -2.04451 & $Z Y J H K_{\mathrm{s}}$ & - \\
\hline b308 & 18:02:27.312 & $-26: 38: 03.12$ & 3.87111 & -2.04447 & $Z Y J H K_{\mathrm{s}}$ & - \\
\hline b309 & $18: 05: 38.232$ & $-25: 21: 47.52$ & 5.33034 & -2.04451 & $Z Y J H K_{\mathrm{s}}$ & - \\
\hline b310 & 18:08:45.480 & $-24: 05: 18.96$ & 6.78962 & -2.04454 & $Z Y J H K_{\mathrm{s}}$ & - \\
\hline b311 & $18: 11: 49.320$ & $-22: 48: 38.52$ & 8.24891 & -2.04449 & $Z Y J H K_{\mathrm{s}}$ & 1 \\
\hline b312 & $18: 14: 50.040$ & $-21: 31: 47.64$ & 9.70816 & -2.04447 & $J H K_{\mathrm{s}}$ & 1 \\
\hline b313 & $17: 25: 32.232$ & $-37: 14: 49.92$ & 350.73753 & -0.95236 & $J H K_{\mathrm{s}}$ & 1 \\
\hline b314 & $17: 29: 34.800$ & $-36: 02: 05.64$ & 352.19625 & -0.95230 & $J H K_{\mathrm{s}}$ & 1 \\
\hline b315 & $17: 33: 30.168$ & $-34: 48: 52.92$ & 353.65504 & -0.95232 & $J H K_{\mathrm{s}}$ & 1 \\
\hline b316 & $17: 37: 18.768$ & $-33: 35: 14.28$ & 355.11368 & -0.95231 & $J H K_{\mathrm{s}}$ & 1 \\
\hline b317 & 17:41:01.104 & $-32: 21: 10.80$ & 356.57248 & -0.95229 & $J H K_{\mathrm{s}}$ & 1 \\
\hline b318 & $17: 44: 37.584$ & $-31: 06: 45.00$ & 358.03121 & -0.95230 & $J H K_{\mathrm{s}}$ & 1 \\
\hline b319 & 17:48:08.616 & $-29: 51: 58.32$ & 359.48996 & -0.95233 & $J H K_{\mathrm{s}}$ & 1 \\
\hline b320 & $17: 51: 34.560$ & $-28: 36: 52.56$ & 0.94861 & -0.95235 & $J H K_{\mathrm{s}}$ & 1 \\
\hline b321 & $17: 54: 55.800$ & $-27: 21: 28.44$ & 2.40742 & -0.95234 & $J H K_{\mathrm{s}}$ & 1 \\
\hline b322 & $17: 58: 12.648$ & $-26: 05: 48.12$ & 3.86612 & -0.95234 & $J H K_{\mathrm{s}}$ & 1 \\
\hline b323 & $18: 01: 25.416$ & $-24: 49: 52.68$ & 5.32477 & -0.95232 & $J H K_{\mathrm{s}}$ & 1 \\
\hline b324 & $18: 04: 34.440$ & $-23: 33: 42.84$ & 6.78355 & -0.95232 & $J H K_{\mathrm{s}}$ & 1 \\
\hline b325 & 18:07:39.984 & $-22: 17: 20.40$ & 8.24226 & -0.95238 & $J H K_{\mathrm{s}}$ & - \\
\hline b326 & $18: 10: 42.288$ & $-21: 00: 45.72$ & 9.70101 & -0.95231 & $J H K_{\mathrm{s}}$ & - \\
\hline b327 & $17: 21: 01.656$ & $-36: 37: 48.00$ & 350.73744 & 0.13984 & $J H K_{\mathrm{s}}$ & 1 \\
\hline b328 & $17: 25: 06.528$ & $-35: 25: 37.20$ & 352.19621 & 0.13989 & $J H K_{\mathrm{s}}$ & 1 \\
\hline b329 & $17: 29: 04.152$ & $-34: 12: 56.52$ & 353.65492 & 0.13987 & $J H K_{\mathrm{s}}$ & - \\
\hline b330 & 17:32:55.008 & $-32: 59: 47.76$ & 355.11368 & 0.13984 & $J H K_{\mathrm{s}}$ & - \\
\hline b331 & $17: 36: 39.504$ & $-31: 46: 13.08$ & 356.57236 & 0.13988 & $J H K_{\mathrm{s}}$ & - \\
\hline b332 & $17: 40: 18.120$ & $-30: 32: 14.28$ & 358.03110 & 0.13982 & $J H K_{\mathrm{s}}$ & - \\
\hline b333 & 17:43:51.192 & $-29: 17: 52.80$ & 359.48985 & 0.13988 & $J H K_{\mathrm{s}}$ & - \\
\hline b334 & $17: 47: 19.128$ & $-28: 03: 10.80$ & 0.94855 & 0.13988 & $J H K_{\mathrm{s}}$ & - \\
\hline b335 & $17: 50: 42.288$ & $-26: 48: 09.36$ & 2.40731 & 0.13985 & $J H K_{\mathrm{s}}$ & - \\
\hline b336 & 17:54:00.984 & $-25: 32: 49.92$ & 3.86610 & 0.13985 & $J H K_{\mathrm{s}}$ & - \\
\hline b337 & $17: 57: 15.528$ & $-24: 17: 14.28$ & 5.32478 & 0.13981 & $J H K_{\mathrm{s}}$ & - \\
\hline
\end{tabular}


Table A.1. continued.

\begin{tabular}{|c|c|c|c|c|c|c|}
\hline $\begin{array}{l}\text { Tile } \\
\text { name }\end{array}$ & $\begin{array}{l}\text { RA (J2000.0) } \\
\text { dd:mm:ss.sss }\end{array}$ & $\begin{array}{c}\text { Dec (J2000.0) } \\
\text { dd:mm:ss.ss }\end{array}$ & $\begin{array}{c}\begin{array}{c}\text { Longitude } \\
\text { degrees }\end{array} \\
\text { deres }\end{array}$ & $\begin{array}{l}\text { Latitude } \\
\text { degrees }\end{array}$ & $\begin{array}{c}\text { Filters } \\
\text { completed }\end{array}$ & $\begin{array}{l}K_{\mathrm{s}} \text { epochs } \\
\text { completed }\end{array}$ \\
\hline b338 & 18:00:26.208 & $-23: 01: 23.16$ & 6.78348 & 0.13983 & $J H K_{\mathrm{s}}$ & - \\
\hline b339 & 18:03:33.336 & $-21: 45: 17.64$ & 8.24226 & 0.13983 & $J H K_{\mathrm{s}}$ & 1 \\
\hline b340 & 18:06:37.152 & $-20: 28: 59.16$ & 9.70099 & 0.13985 & $Z Y J H K_{\mathrm{s}}$ & 1 \\
\hline b341 & $17: 16: 35.472$ & $-36: 00: 07.56$ & 350.73765 & 1.23203 & $Z Y J H K_{\mathrm{s}}$ & 1 \\
\hline b342 & $17: 20: 42.432$ & $-34: 48: 30.24$ & 352.19686 & 1.23205 & $J H K_{\mathrm{s}}$ & 1 \\
\hline b343 & $17: 24: 42.144$ & $-33: 36: 20.88$ & 353.65613 & 1.23203 & $J H K_{\mathrm{s}}$ & 1 \\
\hline b344 & $17: 28: 35.040$ & $-32: 23: 41.64$ & 355.11542 & 1.23207 & $J H K_{\mathrm{s}}$ & 1 \\
\hline b345 & $17: 32: 21.576$ & $-31: 10: 35.04$ & 356.57468 & 1.23205 & $J H K_{\mathrm{s}}$ & 1 \\
\hline b346 & $17: 36: 02.160$ & $-29: 57: 02.52$ & 358.03399 & 1.23203 & $J H K_{\mathrm{s}}$ & 1 \\
\hline b347 & $17: 39: 37.152$ & $-28: 43: 06.24$ & 359.49322 & 1.23206 & $J H K_{\mathrm{s}}$ & 1 \\
\hline b348 & $17: 43: 06.960$ & $-27: 28: 47.64$ & 0.95251 & 1.23203 & $J H K_{\mathrm{s}}$ & 1 \\
\hline b349 & $17: 46: 31.896$ & $-26: 14: 08.52$ & 2.41172 & 1.23201 & $J H K_{\mathrm{s}}$ & 1 \\
\hline b350 & $17: 49: 52.296$ & $-24: 59: 09.96$ & 3.87096 & 1.23204 & $J H K_{\mathrm{s}}$ & 1 \\
\hline b351 & 17:53:08.496 & $-23: 43: 53.40$ & 5.33027 & 1.23202 & $J H K_{\mathrm{s}}$ & - \\
\hline b352 & $17: 56: 20.760$ & $-22: 28: 20.28$ & 6.78955 & 1.23201 & $Z Y J H K_{\mathrm{s}}$ & - \\
\hline b353 & $17: 59: 29.376$ & $-21: 12: 31.68$ & 8.24885 & 1.23202 & $Z Y J H K_{\mathrm{s}}$ & - \\
\hline b354 & 18:02:34.608 & $-19: 56: 29.04$ & 9.70808 & 1.23199 & $Z Y J H K_{\mathrm{s}}$ & - \\
\hline b355 & $17: 12: 13.584$ & $-35: 21: 49.68$ & 350.73827 & 2.32427 & $Z Y J H K_{\mathrm{s}}$ & 1 \\
\hline b356 & $17: 16: 22.488$ & $-34: 10: 45.12$ & 352.19857 & 2.32417 & $Z Y$ & 1 \\
\hline b357 & $17: 20: 24.096$ & $-32: 59: 06.36$ & 353.65894 & 2.32423 & $Z Y$ & - \\
\hline b358 & $17: 24: 18.888$ & $-31: 46: 56.64$ & 355.11924 & 2.32422 & $Z Y$ & - \\
\hline b361 & $17: 35: 26.472$ & $-28: 07: 39.36$ & 359.50024 & 2.32421 & $Z Y$ & - \\
\hline b362 & 17:38:58.008 & $-26: 53: 43.80$ & 0.96059 & 2.32418 & $Z Y J H K_{\mathrm{s}}$ & - \\
\hline b363 & $17: 42: 24.624$ & $-25: 39: 25.92$ & 2.42098 & 2.32420 & $Z Y J H K_{\mathrm{s}}$ & - \\
\hline b364 & $17: 45: 46.632$ & $-24: 24: 47.88$ & 3.88124 & 2.32419 & $Z Y J H K_{\mathrm{s}}$ & - \\
\hline b365 & 17:49:04.368 & $-23: 09: 50.40$ & 5.34158 & 2.32416 & $Z Y J H K_{\mathrm{s}}$ & - \\
\hline b366 & $17: 52: 18.096$ & $-21: 54: 34.92$ & 6.80192 & 2.32418 & $J H K_{\mathrm{s}}$ & 1 \\
\hline b367 & $17: 55: 28.104$ & $-20: 39: 02.88$ & 8.26224 & 2.32419 & $J H K_{\mathrm{s}}$ & 1 \\
\hline b368 & $17: 58: 34.656$ & $-19: 23: 15.00$ & 9.72265 & 2.32423 & $J H K_{\mathrm{s}}$ & 1 \\
\hline b369 & $17: 07: 55.872$ & $-34: 42: 57.24$ & 350.73892 & 3.41637 & $Z Y J H K_{\mathrm{s}}$ & 1 \\
\hline b370 & $17: 12: 06.480$ & $-33: 32: 24.36$ & 352.20083 & 3.41644 & $Z Y J H K_{\mathrm{s}}$ & 1 \\
\hline b371 & 17:16:09.864 & $-32: 21: 16.20$ & 353.66280 & 3.41638 & $Z Y J H K_{\mathrm{s}}$ & - \\
\hline b372 & $17: 20: 06.384$ & $-31: 09: 35.28$ & 355.12466 & 3.41637 & $Z Y J H K_{\mathrm{s}}$ & - \\
\hline b373 & $17: 23: 56.496$ & $-29: 57: 23.04$ & 356.58663 & 3.41642 & $Z Y J H K_{\mathrm{s}}$ & - \\
\hline b374 & $17: 27: 40.584$ & $-28: 44: 42.36$ & 358.04853 & 3.41640 & $Z Y J H K_{\mathrm{s}}$ & - \\
\hline b375 & $17: 31: 19.032$ & $-27: 31: 34.68$ & 359.51046 & 3.41635 & $Z Y J H K_{\mathrm{s}}$ & - \\
\hline b376 & $17: 34: 52.152$ & $-26: 18: 01.44$ & 0.97243 & 3.41644 & $Z Y J H K_{\mathrm{s}}$ & - \\
\hline b377 & $17: 38: 20.328$ & $-25: 04: 05.16$ & 2.43434 & 3.41638 & $Z Y J H K_{\mathrm{s}}$ & - \\
\hline b378 & $17: 41: 43.848$ & $-23: 49: 46.56$ & 3.89631 & 3.41638 & $Z Y J H K_{\mathrm{s}}$ & - \\
\hline b379 & $17: 45: 03.024$ & $-22: 35: 07.44$ & 5.35828 & 3.41637 & $Z Y J H K_{\mathrm{s}}$ & 1 \\
\hline b380 & $17: 48: 18.120$ & $-21: 20: 09.24$ & 6.82020 & 3.41640 & $Z Y J H K_{\mathrm{s}}$ & 1 \\
\hline b381 & $17: 51: 29.424$ & $-20: 04: 53.40$ & 8.28204 & 3.41640 & $J H K_{\mathrm{s}}$ & 1 \\
\hline b382 & $17: 54: 37.224$ & $-18: 49: 20.64$ & 9.74399 & 3.41636 & $J H K_{\mathrm{s}}$ & 1 \\
\hline b383 & $17: 03: 42.216$ & $-34: 03: 30.60$ & 350.73979 & 4.50856 & $Z Y J H K_{\mathrm{s}}$ & 1 \\
\hline b384 & 17:07:54.408 & $-32: 53: 29.40$ & 352.20380 & 4.50857 & $J H K_{\mathrm{s}}$ & 1 \\
\hline b385 & $17: 11: 59.352$ & $-31: 42: 51.12$ & 353.66783 & 4.50858 & $J H K_{\mathrm{s}}$ & 1 \\
\hline b386 & $17: 15: 57.480$ & $-30: 31: 37.92$ & 355.13195 & 4.50860 & $J H K_{\mathrm{s}}$ & 1 \\
\hline b387 & $17: 19: 49.176$ & $-29: 19: 52.68$ & 356.59597 & 4.50856 & $J H K_{\mathrm{s}}$ & 1 \\
\hline b388 & $17: 23: 34.824$ & $-28: 07: 36.84$ & 358.06004 & 4.50857 & $J H K_{\mathrm{s}}$ & 1 \\
\hline b389 & $17: 27: 14.784$ & $-26: 54: 52.56$ & 359.52411 & 4.50859 & $J H K_{\mathrm{s}}$ & 1 \\
\hline b390 & $17: 30: 45.384$ & $-25: 43: 05.52$ & 0.96034 & 4.50854 & $J H K_{\mathrm{s}}$ & 1 \\
\hline b391 & $17: 34: 15.096$ & $-24: 29: 30.12$ & 2.42444 & 4.50852 & $J H K_{\mathrm{s}}$ & 1 \\
\hline b392 & $17: 37: 40.080$ & $-23: 15: 31.32$ & 3.88850 & 4.50858 & $J H K_{\mathrm{s}}$ & 1 \\
\hline b393 & $17: 41: 00.672$ & $-22: 01: 10.92$ & 5.35253 & 4.50853 & $J H K_{\mathrm{s}}$ & - \\
\hline b394 & $17: 44: 17.136$ & $-20: 46: 29.64$ & 6.81666 & 4.50854 & $J H K_{\mathrm{s}}$ & - \\
\hline b395 & $17: 47: 29.736$ & $-19: 31: 29.28$ & 8.28074 & 4.50857 & $Z Y J H K_{\mathrm{s}}$ & - \\
\hline b396 & $17: 50: 38.736$ & $-18: 16: 11.28$ & 9.74476 & 4.50855 & $Z Y J H K_{\mathrm{s}}$ & - \\
\hline
\end{tabular}


1 Departamento Astronomía y Astrofísica, Pontificia Universidad Católica de Chile, Av. Vicuña Mackenna 4860, Santiago, Chile e-mail: rsaito@astro.puc.cl

2 Vatican Observatory, Vatican City State 00120, Italy

3 Department of Astrophysical Sciences, Princeton University, Princeton, NJ 08544-1001, USA

4 Centre for Astrophysics Research, University of Hertfordshire, College Lane, Hatfield AL10 9AB, UK

5 European Southern Observatory, Karl-Schwarzschild-Strasse 2, 85748 Garching, Germany

6 Atacama Large Millimeter Array, Alonso de Córdova 3107, Vitacura, Santiago, Chile

7 Institute of Astronomy, University of Cambridge, Madingley Road, Cambridge CB3 OHA, UK

8 Institute for Astronomy, The University of Edinburgh, Royal Observatory, Blackford Hill, Edinburgh EH9 3HJ, UK

9 European Southern Observatory, Ave. Alonso de Cordova 3107, Casilla 19, 19001 Santiago, Chile

10 Jodrell Bank Centre for Astrophysics, The University of Manchester, Oxford Road, Manchester M13 9PL, UK

11 Astronomy Unit, School of Physics and Astronomy, Queen Mary University of London, Mile End Road, London, E1 4NS, UK

12 Departamento de Física, Universidad de La Serena, Cisternas 1200 Norte, La Serena, Chile

13 Faculdade de Ciências da Universidade de Lisboa, Campo Grande, Edificio C5, 1749-016 Lisboa, Portugal

14 Laboratório Nacional de Astrofísica, Rua Estados Unidos 154, Itajubá-MG, 37504-364, Brazil

15 Instituto de Astronomía Teórica y Experimental, CONICET, Laprida 922, 5000 Córdoba, Argentina

16 Centro de Astro-Ingeniería, Pontificia Universidad Católica de Chile, Av. Vicuña Mackenna 4860, Santiago, Chile

17 INAF - Osservatorio Astronomico di Bologna, via Ranzani 1, 40127 Bologna, Italy

18 Warsaw University Observatory, Al. Ujazdowskie 400-478, Warsaw, Poland

19 Departmento de Astronomía, Universidad de Concepción, Casilla 160-C, Concepción, Chile

20 Departamento de Física y Astronomía, Facultad de Ciencias, Universidad de Valparaíso, Ave. Gran Bretaña 1111, Playa Ancha, Casilla 5030, Valparaíso, Chile

21 Observatorio Astronómico de Córdoba, Universidad Nacional de Córdoba, Laprida 854, 5000 Córdoba, Argentina

22 Consejo Nacional de Investigaciones Científicas y Técnicas, Av. Rivadavia 1917 - CPC1033AAJ - Buenos Aires, Argentina

23 Gemini Observatory, Southern Operations Center, c/o AURA, Casilla 603 La Serena, Chile

24 Department of Astronomy, University of Florida, 211 Bryant Space Science Center PO Box 112055, Gainesville, FL, 32611-2055, USA

25 Instituto de Ciencias Astronómicas, del la Tierra y del Espacio (ICATE-CONICET), Av. España Sur 1512, J5402DSP San Juan, Argentina

26 Universidade de São Paulo, IAG, Rua do Matão 1226, Cidade Universitária, São Paulo 05508-900, Brazil

27 Facultad de Ciencias Astronómicas y Geofísicas, Universidad Nacional de La Plata, and Instituto de Astrofísica La Plata (IALP-CONICET), Paseo del Bosque S/N, B1900FWA, La Plata, Argentina

28 INAF - Astronomical Observatory of Padova, vicolo dell Osservatorio 5, 35122 Padova, Italy
29 Dipartimento di Astronomia, Universitá di Padova, vicolo dell Osservatorio 3, 35122 Padova, Italy

30 Department of Physics, University of Wisconsin-Whitewater, 800 West Main Street, Whitewater, WI 53190, USA

31 Universidade Federal do Rio Grande do Sul, IF, CP 15051, Porto Alegre 91501-970, RS, Brazil

32 Departamento de Astronomía, Universidad de Chile, Casilla 36-D, Santiago, Chile

33 Kavli Institute for Astronomy and Astrophysics, Peking University, Yi He Yuan Lu 5, Hai Dian District, Beijing 100871, PR China

34 Department of Astronomy and Space Science, Kyung Hee University, Yongin-shi, Kyungki-do 449-701, Republic of Korea

35 Max Planck Institute for Astronomy, Königstuhl 17, 69117 Heidelberg, Germany

36 Departamento de Física, Ingeniería de Sistemas y Teoría de la Señal, Universidad de Alicante, Apdo. 99, 03080 Alicante, Spain

37 Mullard Space Science Laboratory, University College London, Holmbury St. Mary, Dorking, Surrey, RH5 6NT, UK

38 Department of Physics, University of Cincinnati, PO Box 210011, Cincinnati, OH 45221-0011, USA

39 School of Physics \& Astronomy, University of Leeds, Woodhouse Lane, Leeds LS2 9JT, UK

40 NASA-Ames Research Center/Bay Area Environmental Research Institute, MS 244-30, Moffett Field, CA 94035, USA

41 UK Astronomy Technology Centre, Royal Observatory, Blackford Hill, Edinburgh EH9 3HJ, UK

42 Instituto de Astrofísica de Canarias, Vía Láctea s/n, E38205 - La Laguna (Tenerife), Spain

43 Departamento de Astrofísica, Universidad de La Laguna, $38206 \mathrm{La}$ Laguna, Tenerife, Spain

44 School of Physics and Astronomy, University of Southampton, Highfield, Southampton, SO17 1BJ, UK

45 Saint Marys University, 923 Robie Street, Halifax, Nova Scotia, Canada

46 Centro de Astrobiologia CSIC - INTA, Carretera Torrejón-Ajalvir $\mathrm{km} \mathrm{4,} \mathrm{28850,} \mathrm{Madrid,} \mathrm{Spain}$

47 Istituto di Astrofisica Spaziale e Fisica Cosmica di Bologna, via Gobetti 101, 40129 Bologna, Italy

48 Service d'Astrophysique - IRFU, CEA-Saclay, 91191 Gif-surYvette, France

49 Instituto de Astronomía y Física del Espacio, Casilla de Correo 67, Sucursal 28, Buenos Aires, Argentina

50 Department of Physics \& Astronomy, Macquarie University, Sydney, NSW 2109, Australia

51 Australian Astronomical Observatory, PO Box 296, Epping, NSW 1710, Australia

52 Departamento de Ciencias Fisicas, Universidad Andres Bello, Av. Republica 252, Santiago, Chile

53 South African Astronomical Observatory, PO Box 9, Observatory 7935, Cape Town, South Africa

54 Hartebeesthoek Radio Astronomy Observatory, PO Box 443, 1740 Krugersdorp, South Africa

55 Aryabhatta Research Institute of Observational Sciences (ARIES), Manora Peak, 263129 Nainital, India

56 The University of Kent, Canterbury, Kent, CT2 7NH, UK

57 Gemini Observatory, Northern Operations Center, 670 N. A'ohoku Place, Hilo, Hawaii, 96720, USA

58 Division of Optical and Infrared Astronomy, National Astronomical Observatory of Japan 2-21-1 Osawa, Mitaka, Tokyo, 181-8588, Japan

59 Departamento de Ingeniería Eléctrica, Pontificia Universidad Católica de Chile, Av. Vicuña Mackenna 4860, Santiago, Chile 